\title{
A SURVEY OF THE HIGH ORDER MULTIPLICITY OF NEARBY SOLAR-TYPE BINARY STARS WITH Robo-AO
}

\author{
Reed L. Riddle ${ }^{1}$, Andrei Tokovinin ${ }^{2}$, Brian D. Mason ${ }^{3}$, William I. HartKopf ${ }^{3}$, Lewis C. Roberts Jr. ${ }^{4}$, \\ Christoph Baranec ${ }^{5}$, Nicholas M. Law ${ }^{6}$, Khanh Bui ${ }^{1}$, Mahesh P. Burse ${ }^{7}$, H. K. Das ${ }^{7}$, Richard G. Dekany ${ }^{1}$, \\ Shrinivas Kulkarni ${ }^{1}$, Sujit Punnadi ${ }^{7}$, A. N. Ramaprakash ${ }^{7}$, and Shriharsh P. TendulKar ${ }^{1}$ \\ ${ }^{1}$ Division of Physics, Mathematics, and Astronomy, California Institute of Technology, Pasadena, CA 91125, USA \\ ${ }^{2}$ Cerro Tololo Inter-American Observatory, Casilla 603, La Serena, Chile \\ ${ }^{3}$ U.S. Naval Observatory, 3450 Massachusetts Avenue, Washington, DC 20392-5420, USA \\ ${ }^{4}$ Jet Propulsion Laboratory, California Institute of Technology, 4800 Oak Grove Drive, Pasadena, CA 91109, USA \\ ${ }^{5}$ Institute for Astronomy, University of Hawai"i at Mānoa, Hilo, HI 96720-2700, USA \\ ${ }^{6}$ Department of Physics and Astronomy, University of North Carolina at Chapel Hill, Chapel Hill, NC 27599-3255, USA \\ ${ }^{7}$ Inter-University Centre for Astronomy \& Astrophysics, Ganeshkhind, Pune, 411007, India \\ Received 2014 July 30; accepted 2014 November 1; published 2015 January 9
}

\begin{abstract}
We conducted a survey of nearby binary systems composed of main sequence stars of spectral types $F$ and $G$ in order to improve our understanding of the hierarchical nature of multiple star systems. Using Robo-AO, the first robotic adaptive optics instrument, we collected high angular resolution images with deep and well-defined detection limits in the Sloan Digital Sky Survey $i^{\prime}$ band. A total of 695 components belonging to 595 systems were observed. We prioritized observations of faint secondary components with separations over $10^{\prime \prime}$ to quantify the still poorly constrained frequency of their subsystems. Of the 214 secondaries observed, 39 contain such subsystems; 19 of those were discovered with Robo-AO. The selection-corrected frequency of secondary subsystems with periods from $10^{3.5}$ to $10^{5}$ days is $0.12 \pm 0.03$, the same as the frequency of such companions to the primary. Half of the secondary pairs belong to quadruple systems where the primary is also a close pair, showing that the presence of subsystems in both components of the outer binary is correlated. The relatively large abundance of $2+2$ quadruple systems is a new finding, and will require more exploration of the formation mechanism of multiple star systems. We also targeted close binaries with periods less than $100 \mathrm{yr}$, searching for their distant tertiary components, and discovered 17 certain and 2 potential new triples. In a subsample of 241 close binaries, 71 have additional outer companions. The overall frequency of tertiary components is not enhanced, compared to all (non-binary) targets, but in the range of outer periods from $10^{6}$ to $10^{7.5}$ days (separations on the order of $500 \mathrm{AU}$ ), the frequency of tertiary components is $0.16 \pm 0.03$, exceeding the frequency of similar systems among all targets (0.09) by almost a factor of two. Measurements of binary stars with Robo-AO allowed us to compute first orbits for 9 pairs and to improve orbits of another 11 pairs.
\end{abstract}

Key words: binaries: close - binaries: general - instrumentation: adaptive optics - stars: formation techniques: high angular resolution

Supporting material: machine-readable tables

\section{INTRODUCTION}

Statistics of hierarchical stellar systems provide important clues to star formation mechanisms that are still actively researched and debated. Current theories give contradictory predictions about hierarchical multiples (for example, $N$-body dynamics versus hydrodynamical simulations produce different multiplicity fractions); so far, none of them is capable of modeling the distributions of periods, mass ratios, and hierarchies seen in observations.

As periods and separations of stellar pairs vary by several orders of magnitude, comprehensive coverage of the parameter space can be achieved only by combining complementary observing techniques, and only for the nearest stars. However, the number of objects in the best-studied $25 \mathrm{pc}$ sample of Raghavan et al. (2010) is too small for deriving meaningful statistics of triple and higher-order multiples. For this purpose, a larger, distance-limited sample of $\sim 5000 \mathrm{~F}$ and $\mathrm{G}$ dwarfs within $67 \mathrm{pc}$ of the Sun was constructed from the Hipparcos catalog; it is called FG-67 hereafter.

The sample definition and the database are presented in Tokovinin (2014). The census of companions to primary stars in FG-67 is fairly complete over the whole range of periods, with an overall detection probability of about $80 \%$, except for lowmass companions at separations from $0{ }^{\prime} 1$ to $20^{\prime \prime}$ (see details in the above paper). Such companions can be discovered by high-contrast imaging with adaptive optics (AO). Secondary components can be close pairs as well, but discovery of subsystems in the secondary components is more problematic, with average detection probability (before this survey) of only about 15\%; most subsystems in the secondaries are presently missed. If they are as frequent as subsystems in the primaries, the actual number of stellar hierarchies in the FG-67 sample is substantially larger than known today.

It is important to test the frequency of subsystems in the secondaries for constraining mechanisms of multiple-star formation. If chaotic $N$-body dynamics is the dominant mechanism, subsystems in the secondaries should be much less common than in the primaries because, in triple encounters, the lightest star is usually ejected while the two most massive stars pair in a binary. On the other hand, the hydrodynamic simulations of Bate (2012) predict a large fraction of multiple systems, including $2+2$ quadruples (i.e., two close pairs in a wide outer binary).

Recent advances in observational techniques have substantially improved our knowledge of the distribution of binary periods and mass ratios, as reviewed by Duchêne \& Kraus (2013). 


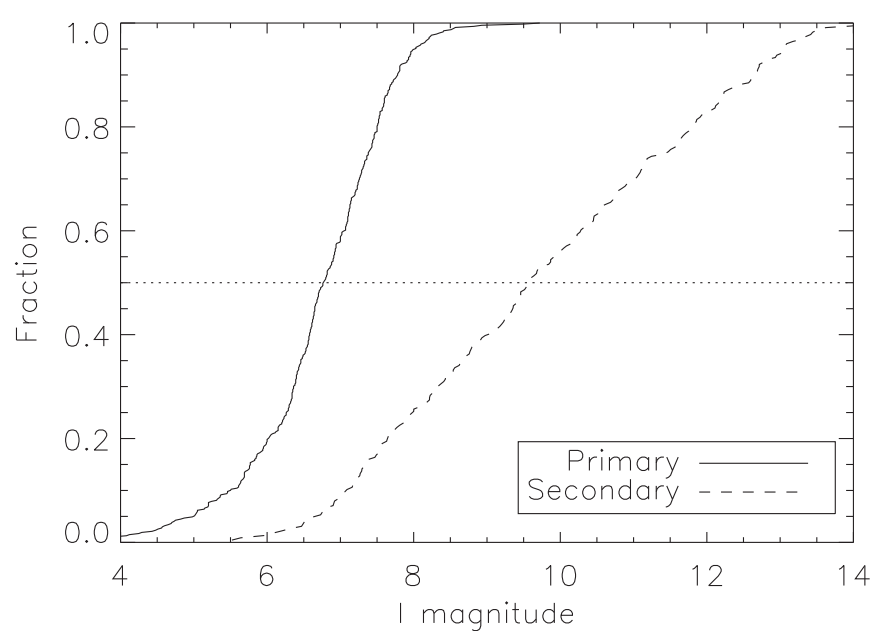

Figure 1. Cumulative histograms of the $I_{C}$ magnitudes of components observed with Robo-AO.

Lucky imaging has been used to survey large samples (Ginski et al. 2012; Janson et al. 2012; Jodar et al. 2013). Yet, the emphasis is almost always placed on binaries, leaving higher-order hierarchies aside or addressing them casually; a rare exception is the work of Law et al. (2010) who observed a higher than expected high-order multiplicity of $\mathrm{M}$ dwarf stars.

This survey aims at complementing the statistics of hierarchical systems in the FG-67 sample by imaging with Robo-AO (Baranec et al. 2014). Our main goal is to constrain the multiplicity of secondary components, most of which so far had no high-resolution imaging data. The second goal is to search for additional companions around primary targets that are themselves known close binaries. Here we explore the poorly covered part of the parameter space at separations on the order of an arcsecond, searching for low-mass tertiary components. In short, we focus on binaries (both wide and close) and quantify the frequency of additional companions.

We begin with a brief characterization of the surveyed sample in Section 2. Observations with Robo-AO and data processing are covered in Section 3, with data tables described in Section 4. In Section 5 the results of this survey are presented, and Section 6 is the summary.

\section{THE TARGET SAMPLE}

The targets for this survey were selected from the FG-67 database (Tokovinin 2014). We observed known and suspected binary stars and tried to constrain the frequency of additional components in those systems; high priority was placed on distant secondary components in wide binaries. The selection criteria were separations of $>10^{\prime \prime}$ and a declination north of $-15^{\circ}$. Some brighter secondaries are in the Hipparcos catalog, and therefore "screened" for companions by that space mission (but not as deeply as with Robo-AO). The majority, however, are rather faint. Many of those secondaries were discovered only recently by dedicated programs (Tokovinin 2011; Tokovinin \& Lépine 2012).

The second-priority list contains mainly targets that are binaries with periods $P<100 \mathrm{yr}$ (separation $\sim 0^{\prime} .5$ at $50 \mathrm{pc}$ distance). The rationale for restricting the periods is related to dynamical stability: the semi-major axis of the outer orbit should be at least three times larger than that of the inner orbit, and should match well the discovery space of Robo-AO. Harrington (1992) quantified a value of three for the ratio of
Table 1

Robo-AO Observation Parameters

\begin{tabular}{lc}
\hline \hline Observation date range & 2012 Aug 31 to 2013 August 21 \\
\hline Targets observed & 695 \\
Total observing time & $29 \mathrm{hr}$ \\
Observation filters & SDSS $i^{\prime}$, LP600 \\
Exposure time & $60 \mathrm{~s}$ \\
Typical FWHM resolution & $0^{\prime \prime} .12$ to $0^{\prime \prime} .15$ \\
Typical contrast ratio at 0.5 & $\Delta \sim 5 \mathrm{mag}$. \\
\hline
\end{tabular}

periastron of the outer binary to apastron of the inner binary as the critical factor for long-term stability (assuming equal masses). The program was later complemented with observations of additional resolved visual binaries with separations from $0^{\prime \prime} .2$ to $2^{\prime \prime}$, and $P>100 \mathrm{yr}$. Here the chances of discovering wider tertiary components are small. The chances of resolving a secondary into a hereto unknown close pair are also small. However, observing those stars (mostly resolved) was useful for improving their orbits and for confirming some uncertain visual pairs that were resolved only once in the past and never confirmed since. The program included repeated observations of several known binaries as calibrators for data quality control.

We observed most, but not all, components from the original lists: 239 secondaries ( 212 of those with good quality), 354 close pairs, and 102 resolved binaries. Some secondaries turned out to be too faint. Figure 1 presents the distribution of $I_{C}$ (Cousins $I$ ) magnitudes of our primary targets (van Leeuwen 2007) and of their secondary components. The latter are derived from their $J$ magnitudes in the Two Micron All Sky Survey (2MASS) catalog (Cutri et al. 2003), assuming that the stars are on the main sequence. All secondaries with useful data have $I_{C}<13.5 \mathrm{mag}$. The median masses of primary and secondary components estimated from their absolute magnitudes are 1.22 and $0.71 \mathcal{M}_{\odot}$, respectively; $80 \%$ of secondary masses are between 0.31 and $1.04 \mathcal{M}_{\odot}$.

\section{OBSERVATIONS AND DATA REDUCTION}

The instrument used for this survey was Robo-AO, the first robotic laser guide star AO system (Baranec et al. 2014), which is designed to operate automatically on 1-3 m class telescopes in order to undertake high efficiency observing programs (e.g., large surveys). The prototype Robo-AO instrument is currently deployed on the Palomar Observatory 60 inch $(1.5 \mathrm{~m})$ telescope (P60).

As a fully automated system, Robo-AO is unique in its ability to observe targets at both high resolution and high cadence. Robo-AO uses Rayleigh scattering from a UV laser focused at $10 \mathrm{~km}$ from the telescope as the wavefront reference, and generates images at the diffraction limit of the P60 (0'.12-0'.15 FWHM) with Strehl ratios of $10 \%-25 \%$ in the visible filters used for this survey. The AO system corrects the high order wavefront aberrations with automated software that operates at a rate of $1.2 \mathrm{kHz}$, sharpening the instantaneous point-spread function (PSF) across the science camera field of view (FOV). A bright star within the FOV is still required to correct the tiptilt motion; this is achieved in the automated data processing software (Section 3.2). Typically, Robo-AO requires a star brighter than $m \approx 16$ mag with a broadband filter to apply the tip-tilt correction successfully. Instrument parameters for this survey are listed in Table 1. 


\subsection{Observations}

Baranec et al. (2013) and Baranec et al. (2014) describe the operation and the science instrumentation of the Robo-AO system. The output of the science camera raw data are image cubes composed of $1024 \times 1024$ pixel image frames; a total of 516 frames were gathered during each $60 \mathrm{~s}$ exposure, which were then combined into a single image for further analysis by the automated data processing software. Almost all targets were observed with the Sloan Digital Sky Survey (SDSS) $i^{\prime}$ filter (York et al. 2000) with a central wavelength of $754 \mathrm{~nm}$ and a FWHM bandpass of $119 \mathrm{~nm}$. Some fainter targets were observed with a long pass $600 \mathrm{~nm}$ filter (LP600) that transmits all wavelengths longer than $600 \mathrm{~nm}$ to the quantum efficiency cutoff of the detector.

The pointing error of the P60 telescope with Robo-AO mounted is on the order of $10^{\prime \prime}$, and can vary between observing runs depending on telescope balance and adjustments. With a $44^{\prime \prime}$ FOV, and no clear way to select one target over another in the stellar field, Robo-AO does not attempt to recenter the target automatically in fear of selecting the wrong star and moving the science target out of the field. This effect causes a large variation in the placement of the target star in the image frame; a planned upgrade to the Robo-AO software and continuing improvements to the P60 will minimize this effect. All observations were checked to confirm that the science target was captured in the $\mathrm{CCD}$ frame. The consequence for this survey is that target placement is across the entire FOV, and targets sometimes ended up uncomfortably close to the edge of the frame, limiting the observable area around the target. When necessary, stars affected were reobserved to capture a larger area and allow a better examination for companions.

Robo-AO can observe targets at a rate of $\sim 20$ per hour, with an intelligent queue system selecting the best object to observe at a given time (Riddle et al. 2014). The Robo-AO queue system interleaves several different science programs through a single night. Observations of this survey were spread over a year (2012 July to 2013 August) to gather targets at all hours of right ascension. The entire 695 targets of this survey used 29 total hours of observing time (this time also included calibration binary observations and reobservations). Robo-AO is ideally suited to a large survey such as this one, and is currently the only $\mathrm{AO}$ system that can observe this many targets in such a short time (Terziev et al. 2013; Law et al. 2014a).

\subsection{Automated Data Processing}

The data were processed by automated reduction software developed for Robo-AO image alignment (see Law et al. 2014b, 2014a for the details). Each individual frame is corrected for bias, dark current and flat field using standard calibration data, and the frames are then run through the software to co-add them and create a final high resolution image oversampled to $2048 \times 2048$.

Stars in each output image were identified visually and their approximate centers were marked. Relative astrometry and photometry of wide pairs where the images do not overlap was done by fitting the scaled and shifted image of the brightest component to the secondary within 10 pixel radius from the image center. For closer (overlapping) binaries we used blind deconvolution, as described by Tokovinin et al. (2013). However, most of these "blind" measures are superseded by the results of speckle processing.

\subsection{Binary-star Measurement by Speckle Processing}

Speckle processing is complementary to the blind deconvolution of images, as it delivers diffraction-limited resolution even for low-Strehl data, when the instantaneous PSF has multiple spikes (speckles). The algorithm takes care of the multispeckle PSF and works well even at low flux, when the selection of the brightest pixel for recentering lucky images is compromised by the photon noise. Moreover, the power spectrum (or auto-correlation function, ACF) is proportional to the square of the signal and, therefore, it automatically assigns high weight to sharp images. For widely spaced binaries, speckle processing is done for each component individually to detect close subsystems.

Subframes of $256 \times 256$ original (unbinned) pixels centered on each component were selected from the data cubes. The background was estimated as median signal in each column of the full image and subtracted, removing the systematic bias pattern along the CCD lines. No flat-field correction was applied. The average power spectrum was calculated on the 256 frame cube for each selected component. In parallel, an image was produced from these data by recentering on the selected component and weighting individual frames with the maximum intensity in each frame. The weight is therefore proportional to the image sharpness. These auxiliary images are not used for astrometry or photometry, but are helpful for verifying companion detection and for resolving the $180^{\circ}$ ambiguity of position angle inherent to the classical speckle processing.

The algorithm of speckle data processing and binary-star measurement used here is adapted from the work of Tokovinin et al. (2010). Figure 2 illustrates the case of a close 0!' 12 pair near the diffraction limit. Extracting astrometry and photometry from the image appears problematic, whereas the "fringes" in the power spectrum are very clear and constrain the position and magnitude difference $\Delta m$.

For binaries with nearly equal components, the pipeline image contains an "antipode" because the brightest pixel on which the frames are recentered may belong to either binary component. In this case the relative photometry derived from the image is wrong (although correctable), but the speckle processing delivers the correct $\Delta m$. It also increases the resolution on distant secondary components where the full-frame images suffer from the tilt anisoplanatism. On the other hand, if the secondary component is very faint, the pipeline technique of recentering on the bright primary works much better than speckle processing of the secondary alone. We quantify the strength of the speckle signal by its ratio to the level of photon noise at a spatial frequency two times less than the cutoff frequency, and consider all measures resulting from the "weak" data to be uncertain.

Speckle processing and imaging are truly complementary. In the final step, we combine binary-star measures from those two processing techniques. For binaries with $\rho<2^{\prime \prime}$ the speckle measures are preferred (with a few exceptions where the speckle processing failed), for wider pairs measures from the images are retained. Comparison of repeated measures of the same pairs by speckle and blind deconvolution shows their excellent agreement and gives an idea of the precision. Table 2 lists average values and rms scatter for two sample binaries measured by both methods several times.

\subsection{Detection Limits}

Limits for detecting companions in the pipeline images are evaluated in the standard way. Fluctuation of the signal in 


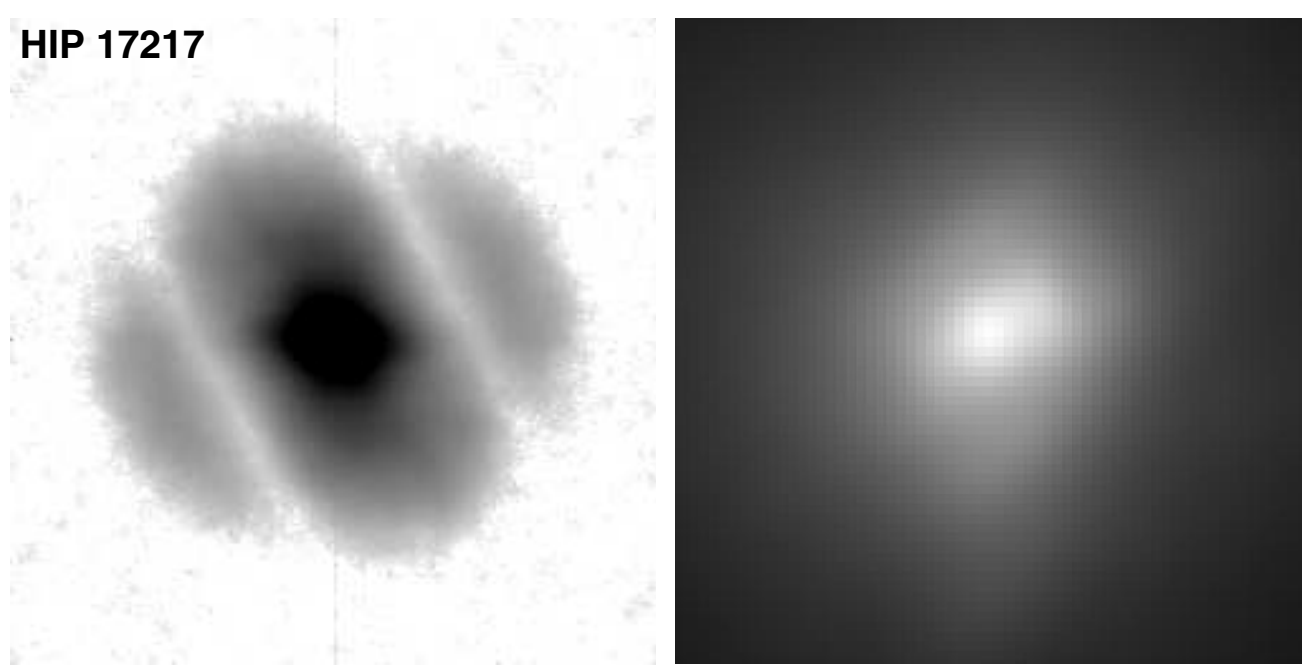

Figure 2. Power spectrum (left, inverse logarithmic scale from $10^{-2}$ to $10^{-7}$ ) and lucky image (right) of HIP 17217 (separation $0^{\prime \prime} 118, \Delta m=0.36$ ).

Table 2

Comparison between Speckle and Blind Deconvolution

\begin{tabular}{|c|c|c|c|c|c|c|c|c|}
\hline \multirow[t]{2}{*}{ HIP1 } & \multirow[t]{2}{*}{ Discoverer } & \multirow[t]{2}{*}{$N_{\text {obs }}$} & \multicolumn{3}{|c|}{ Speckle (mean, rms) } & \multicolumn{3}{|c|}{ Blind (mean, rms) } \\
\hline & & & $\theta$ & $\rho$ & $\Delta m$ & $\theta$ & $\rho$ & $\Delta m$ \\
\hline \multirow[t]{2}{*}{20472} & STF 535 & 9 & 271.15 & 1.130 & 0.98 & 271.18 & 1.129 & 1.04 \\
\hline & & & 0.32 & 0.002 & 0.10 & 0.30 & 0.002 & 0.06 \\
\hline \multirow[t]{2}{*}{23100} & COU 1525 & 8 & 293.55 & 1.833 & 3.36 & 293.56 & 1.832 & 3.32 \\
\hline & & & 0.21 & 0.007 & 0.05 & 0.24 & 0.007 & 0.12 \\
\hline
\end{tabular}

annular zones surrounding the star are computed and it is assumed that the companions brighter than $5 \sigma$ are detectable. The same method is applied to the ACFs in the speckle processing. The two estimates of the detection limits obtained in this way are very similar, with the speckle limits being normally a little deeper (Figure 3). Depending on variable $\mathrm{AO}$ correction and target brightness, the individual detection limits vary substantially. For each target, we list the best (deepest) limits at three characteristic separations of 0.15 , 0 ".8 and 2". 1 , selecting the best of two alternative processing techniques. When the target was observed several times, the deepest detection limits are reported in the table of final results.

All of the survey primary targets are bright, but some secondary components present a problem as they are too faint to provide a useful signal in the power spectrum. Their pipeline images can show a bright 1 pixel spike at the center surrounded by a fuzzy halo; this is created by recentering on the photon events, rather than on the brightest speckle, so the resolution is lost. Detection limits computed formally from such images are over-estimated. However, we prefer to keep such weak data in the final table, despite this obvious caveat, because they still contain some information on the duplicity.

\subsection{Calibration and Distortion Correction}

The reimaging system of the Robo-AO instrument contains a double optical relay with off-axis parabolic mirrors. Such relays are known to introduce quadratic image distortion. In the case of Robo-AO, the distortion, as reported by the optical design, is quite strong. It is directed along the visible CCD columns and displaces all sources down by as much as 26 pixels in the corners.

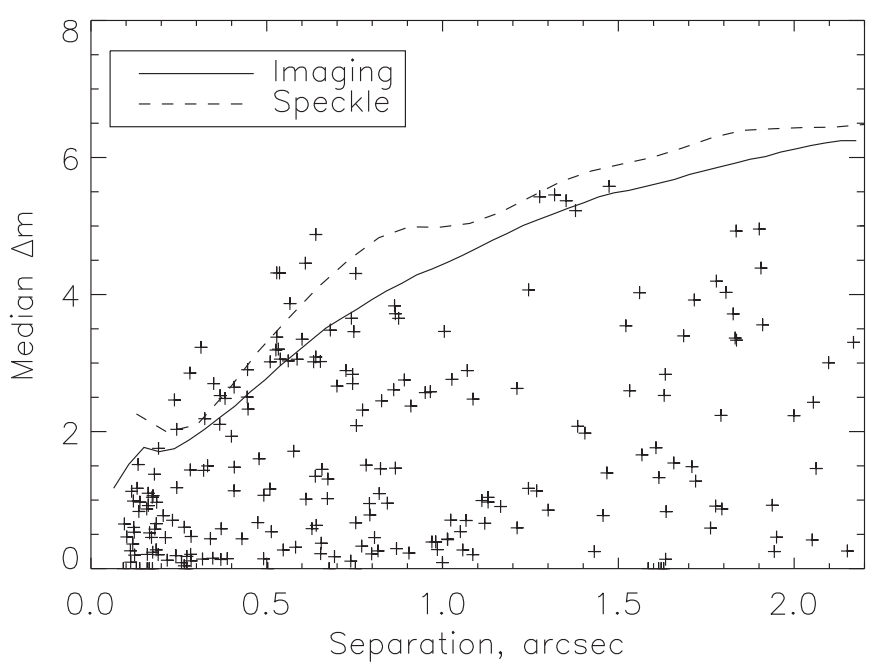

Figure 3. Median detection limit $\Delta m(\rho)$ determined on the images (full line) and on the speckle ACFs (dashed line). Measured pairs are plotted as crosses.

Independently of the optical design, the distortion in Robo-AO was mapped on the sky using the image of the globular cluster M 15 (S. R. Hildebrandt, private communication, 2012). The pixel scale in the $X$-direction was found to be 43.74 mas, while the scale in $Y$ was slightly different. The orientation is such that the $+Y$ axis of the detector points at a position angle 23.9 from North and the $+X$ axis points East (the image has mirror orientation). The quadratic distortion agrees with the optical-design data and is directed along $Y$.

If we express the actual $X, Y$ star coordinates in the image in the "small" over-sampled pixels of the automatic pipeline reduced image, the undistorted coordinate $Y^{\prime}$ is determined as

$$
Y^{\prime}=Y-0.0299 \Delta Y+C_{X}(\Delta X)^{2}+C_{Y}(\Delta Y)^{2},
$$

where $\Delta X$ and $\Delta Y$ are counted from the pipeline image center at $(1024,1024)$ and the coefficients are $C_{Y}=-2.5410^{-5}$, $C_{X}=-2.5510^{-5}$. The linear term in $Y$ corresponds to the modified vertical scale. It is caused by the mismatch between the optical and CCD centers combined with the quadratic distortion.

This distortion substantially affects the measurements of even close binaries because the CCD lines projected on the sky are parabolic. The distortion-induced tilt of the lines reaches 
Table 3

List of Observed Components and Detection Limits

\begin{tabular}{|c|c|c|c|c|c|c|c|c|c|c|}
\hline HIP1 & Comp. & $\begin{array}{c}\text { RA (2000) } \\
(\text { h m s })\end{array}$ & $\begin{array}{c}\operatorname{Dec}(2000) \\
\left({ }^{\prime} \prime \prime \prime\right)\end{array}$ & $\begin{array}{c}V \\
(\mathrm{mag})\end{array}$ & $\begin{array}{l}\text { Sep. } \\
\left({ }^{\prime \prime}\right)\end{array}$ & $\begin{array}{l}\text { Epoch } \\
+2000\end{array}$ & $\begin{array}{l}0^{\prime \prime} .15 \\
(\mathrm{mag})\end{array}$ & $\begin{array}{c}0.8 \\
(\mathrm{mag})\end{array}$ & $\begin{array}{c}2^{\prime \prime} .1 \\
(\mathrm{mag})\end{array}$ & $\begin{array}{c}\rho_{\max } \\
\left({ }^{\prime \prime}\right)\end{array}$ \\
\hline 179 & A & 000216.68 & -132426.8 & 6.90 & 0.0 & 13.5730 & 2.7 & 5.2 & 6.3 & 13.9 \\
\hline 223 & A & 000247.17 & +020748.5 & 7.17 & 0.0 & 12.7049 & 2.1 & 4.9 & 5.7 & 17.5 \\
\hline 394 & A & 000453.76 & +343935.3 & 6.09 & 0.0 & 12.7020 & 1.7 & 5.1 & 6.7 & 15.1 \\
\hline 493 & $\mathrm{D}$ & 000554.75 & +181406.0 & 7.47 & 0.0 & 12.6747 & 1.2 & 4.5 & 6.9 & 11.8 \\
\hline 601 & A & 000718.15 & +205754.3 & 9.14 & 0.0 & 12.7050 & 1.8 & 4.2 & 4.7 & 17.9 \\
\hline 754 & B & 000913.92 & +251638.9 & 11.46 & 29.6 & 12.6747 & 1.6 & 4.7 & 6.8 & 12.2 \\
\hline
\end{tabular}

(This table is available in its entirety in machine-readable form.)

5.6 in the corners of the CCD. We correct the geometric distortion using the pixel coordinates of each source in the images. Equation 1 is applied to each component of a binary. The parameters $\rho, \theta$ are recalculated from the difference of undistorted pixel coordinates $X, Y^{\prime}$. In some instances the angles of close binaries are changed by as much as $2^{\circ}$ after distortion correction. The scatter of relative positions of binaries measured several times is reduced after distortion correction, while the relative positions of wide pairs became closer to their positions derived from 2MASS.

\subsection{Caveats}

As with any observing program, there were issues with some of the data collected that required some extra effort to make it useful. Each pipeline image was examined manually to find and remove erroneous measurements or detections; a graphical IDL tool was created to do this, and also used to mark the components and fit wide binaries. Owing to the $\mathrm{P} 60$ pointing errors, some images were empty, or the targets were found close to the edge of the FOV (see Section 3.1).

The quality of AO compensation, Strehl ratio, and the width of the PSF depended on the seeing conditions and varied substantially. As a consequence, the depth of companion detection was also variable. In some instances we detected new companions in the good-quality images, but missed them in the poor ones. The PSF had a persistent structure ("static speckle") that could be mistaken for a companion. This structure also depended on the AO compensation quality and, possibly, on other factors. Fortunately, there were always other stars observed in the same conditions so the PSF could be compared in order to verify suspicious detections.

In a few cases, the pipeline algorithm occasionally centered a frame on a bright spike caused by cosmic rays, while the remaining frames were centered correctly. This produced a false satellite that could be mistaken for a new companion. Also, several faint satellites caused by internal reflections in the optics are always visible around bright $(V \lesssim 3 \mathrm{mag})$ targets. These reflections effectively reduce the dynamic range of companion detection. We were able to reduce some images where the primary component was saturated. Such data are still useful for detecting wide faint companions. Relative astrometry and photometry of such binaries was done by fitting PSF within an annulus, i.e., excluding the central saturated PSF core from the fit.

The accuracy of the measured positions of wide binaries is less than for the close ones. This is likely caused by the residual tilt anisoplanatism: the differential tilt between the components caused by high-altitude turbulence is not completely averaged during the short $60 \mathrm{~s}$ exposure.
In many cases, we were able to reobserve targets after the data analysis showed that the data were not sufficient; the ability of Robo-AO to observe quickly and efficiently allowed many objects to be added to the final analysis that would have not been available otherwise.

\section{OBSERVING DATA TABLES}

The list of all components successfully observed in this program is given in Table 3. The list contains 695 components belonging to 595 systems. Its columns contain the HIP1 designation (which is the Hipparcos number of the primary component in each system, and is used as the main identifier through this paper), component designation, equatorial coordinates of the observed component, its $V$ magnitude, and its separation from the primary (these data are taken from the main database of the FG-67 sample). Secondary components have non-zero separations. The following columns list the date of observation and detection limits at separations of $0.15,0.8$, and $2^{\prime \prime}$. 1 . The last column gives the distance from the star to the nearest frame border, indicating the maximum separation of its detectable satellites.

Measurements of resolved doubles are given in Table 4. Each pair is identified by its WDS code (Mason et al. 2001b), discoverer designation, and component designation. In column 3, the HIP1 number of the primary star in each system is given. Following that are the date and number of observations (multiple observations made in the same filter within $0.2 \mathrm{yr}$ are averaged), position angle (degrees), position angle error (degrees), separation (arcseconds), separation error(milliarcseconds), and magnitude difference. The errors of position angle and separation are computed as errors of the mean in the case of averaged data, otherwise estimated from the speckle processing, or listed as zero for PSF fitting of wide pairs. Uncertain measures (weak speckle signal or blind deconvolution) are marked by colons after $\Delta m$. We also mark the bandpass when it differs from $i^{\prime}$ (the LP600 filter is denoted by $w$ ). The last three columns give deviations in position angle and separation from the orbits, when available.

\section{RESULTS}

\subsection{Newly Resolved Systems}

The list of newly resolved binary companions (including unrelated background stars) is extracted from the main table and presented separately in Table 5; they are given the "discoverer code" RAO in the WDS; a space is added between discoverer codes and component designations to make it clear they are not the same (i.e., component designations such as Aa and discoverer codes such as RAO 20 are parallel and must not 

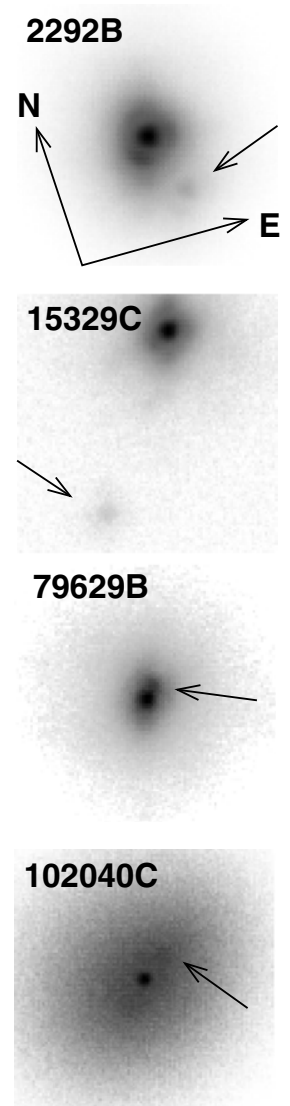

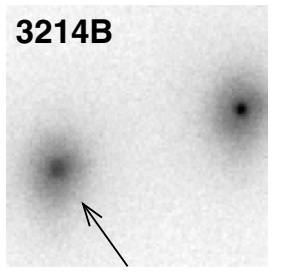

$29860 E$

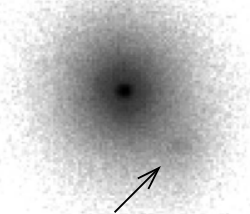

85042B

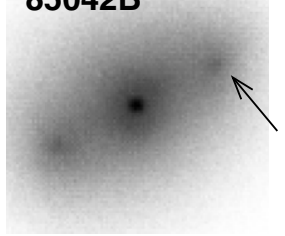

103455

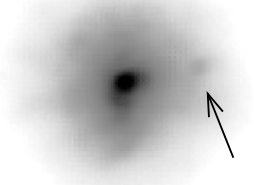

$3540 B$

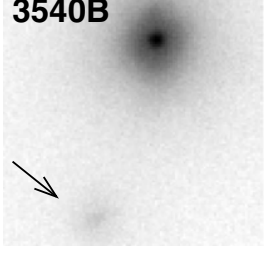

48273B

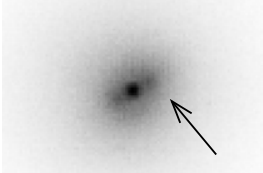

91120B

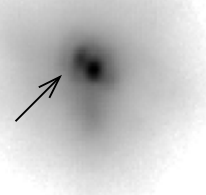

109361

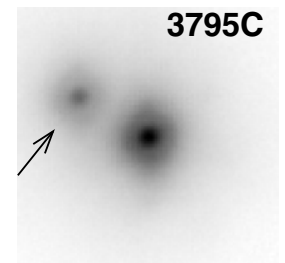

69322B

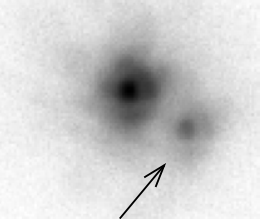

99232

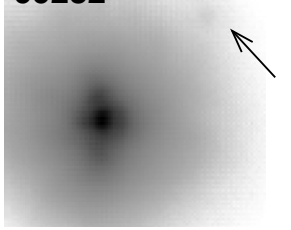

112935B

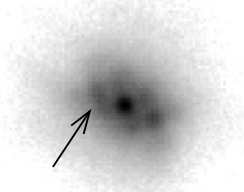

12189B

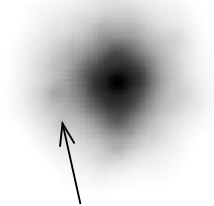

75676B

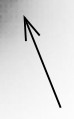

101234

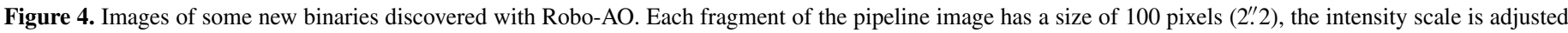
to highlight the companions, indicated by arrows. The images are displayed without rotation and labeled by HIP numbers.

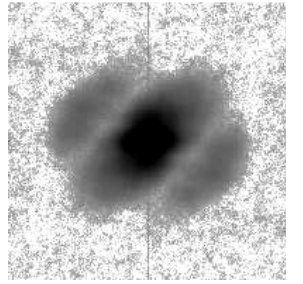

91120B

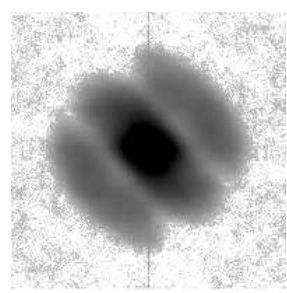

16329B

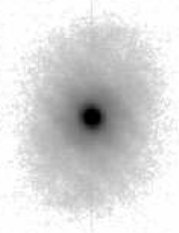

49638

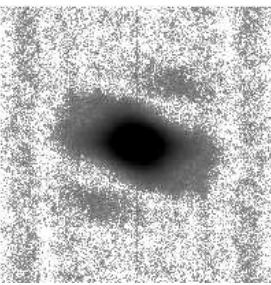

$79629 B$

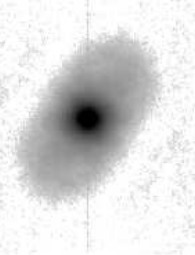

110574

Figure 5. Power spectra of some newly resolved close binaries. Primaries are identified by their HIP1 number.

Table 4

Measurements of Binary Stars with Robo-AO

\begin{tabular}{|c|c|c|c|c|c|c|c|c|c|c|c|c|}
\hline $\begin{array}{l}\text { WDS } \\
(2000)\end{array}$ & $\begin{array}{l}\text { Discoverer } \\
\text { Designation }\end{array}$ & HIP1 & $\begin{array}{l}\text { Epoch } \\
+2000\end{array}$ & $N$ & $\begin{array}{c}\theta \\
(\mathrm{deg})\end{array}$ & $\begin{array}{c}\sigma_{\theta} \\
(\operatorname{deg})\end{array}$ & $\begin{array}{c}\rho \\
\left({ }^{\prime \prime}\right)\end{array}$ & $\begin{array}{c}\sigma \rho \\
(\mathrm{mas})\end{array}$ & $\begin{array}{c}\Delta m \\
(\mathrm{mag})\end{array}$ & $\begin{array}{c}{[O-C]_{\theta}} \\
\quad(\operatorname{deg})\end{array}$ & $\begin{array}{c}{[O-C]_{\rho}} \\
\left({ }^{\prime \prime}\right)\end{array}$ & Ref. \\
\hline $00028+0208$ & BU $281 \mathrm{AB}$ & 223 & 12.7049 & 1 & 160.8 & 0.0 & 1.567 & 0.5 & 1.66 & & & \\
\hline \multirow[t]{2}{*}{$00057+4549$} & STT $547 \mathrm{AB}$ & 473 & 12.7723 & 3 & 186.9 & 0.2 & 6.026 & 42.8 & 0.09 & -0.3 & 0.001 & 1 \\
\hline & & & 13.0478 & 2 & 186.4 & 0.1 & 5.758 & 68.7 & 0.16 & -0.9 & -0.266 & 1 \\
\hline $00057+4549$ & POP 217 AP & 473 & 13.0478 & 2 & 325.5 & 0.3 & 13.140 & 49.4 & 5.03 & & & \\
\hline $00073+2058$ & HDS 12 & 601 & 12.7050 & 1 & 6.7 & 0.1 & 1.864 & 2.0 & $2.38:$ & & & \\
\hline $00251+4803$ & HDS 56 AB & 1987 & 12.7020 & 1 & 279.3 & 0.1 & 0.324 & 0.5 & 2.19 & -0.8 & -0.016 & 2 \\
\hline $00261-1123$ & YR 4 & 2066 & 13.5730 & 1 & 176.8 & 0.0 & 0.376 & 0.0 & $3.12:$ & & & \\
\hline $00271-0753$ & A $431 \mathrm{AB}$ & 2143 & 13.5730 & 1 & 157.6 & 0.1 & 0.166 & 0.3 & 0.00 & -5.0 & -0.019 & 3 \\
\hline
\end{tabular}

References. (1) Kiyaeva et al. (2001); (2) Cvetkovic (2011); (3) Scardia (1981).

(This table is available in its entirety in machine-readable form.)

be confused). Some binaries were measured several times, confirming the measured resolution. Images of new close pairs are presented in Figure 4. The closest pairs are detected in the speckle processing by examining the power spectra; these cases are illustrated by Figure 5. The new wide physical pairs are confirmed by comparing the measured positions of the components with their position in the 2MASS catalog, and by considering additional information such as magnitude, 
Table 5

New Pairs Resolved with Robo-AO

\begin{tabular}{|c|c|c|c|c|c|c|c|}
\hline $\begin{array}{l}\text { WDS } \\
(2000)\end{array}$ & HIP1 & $\begin{array}{l}\text { Discoverer } \\
\text { Designation }\end{array}$ & $N$ & $\begin{array}{c}\theta \\
(\mathrm{deg})\end{array}$ & $\begin{array}{l}\rho \\
(")\end{array}$ & $\begin{array}{c}\Delta m \\
(\mathrm{mag})\end{array}$ & Status, remarks \\
\hline $00293-0555$ & 2292 & RAO $1 \mathrm{BC}$ & 5 & 166.7 & 0.53 & 3.3 & $\mathrm{P}$ (fixed for $1 \mathrm{yr}$ ), $\mathrm{B}=\mathrm{HIP} 2350$ \\
\hline $00346+6235$ & 2717 & $\mathrm{RAO} 2 \mathrm{BC}$ & 1 & 67.2 & 3.55 & 4.3 & P (2MASS image) \\
\hline $00346+6235$ & 2717 & RAO 2 BD & 1 & 302.5 & 3.180 & 6.85 & O (crowded field, non-hierarchical) \\
\hline $00409+3107$ & 3214 & $\mathrm{RAO} 3 \mathrm{Ba}, \mathrm{Bb}$ & 3 & 275.6 & 1.629 & 0.0 & $\mathrm{P}$ (bright, 2MASS image, fixed for $1 \mathrm{yr}$ ) \\
\hline $00452+0015$ & 3540 & $\mathrm{RAO} 4 \mathrm{BC}$ & 1 & 223.1 & 1.521 & 3.5 & P (confirmed with P200, low crowding) \\
\hline $00487+1841$ & 3795 & $\mathrm{RAO} 5 \mathrm{Ca}, \mathrm{Cb}$ & 1 & 322.4 & 0.674 & 1.3 & P (bright \& close, low crowding) \\
\hline $00515+5630$ & 4016 & RAO $38 \mathrm{BC}$ & 1 & 270.4 & 16.999 & 3.97 & O (crowded field, 2MASS position) \\
\hline $00515+5630$ & 4016 & RAO $38 \mathrm{CD}$ & 1 & 82.5 & 1.720 & 1.28 & $\mathrm{O}$ ? $\mathrm{CD}$ is unrelated to $\mathrm{B}$ \\
\hline $01027+0908$ & 4878 & RAO $39 \mathrm{AB}$ & 1 & 36.2 & 2.927 & 6.57 & $\mathrm{O}$ ? (different position in $1 \mathrm{yr}$ ) \\
\hline $01027+0908$ & 4878 & RAO 39 AC & 1 & 147.0 & 32.155 & 6.33 & O (2MASS position, small PM) \\
\hline $01075+4116$ & 5276 & $\mathrm{RAO} 40 \mathrm{AB}$ & 2 & 335.4 & 6.167 & 6.4 & P (2MASS position, conf. with P200) \\
\hline $01080+3915$ & 5313 & $\mathrm{RAO} 41 \mathrm{AB}$ & 1 & 244.7 & 26.24 & 4.4 & $\mathrm{P}$ (2MASS position, color) \\
\hline $01253+0128$ & 6653 & RAO 6 AB & 3 & 204.5 & 1.827 & 4.2 & $\mathrm{P}$ (low crowding, red color) \\
\hline $01409+6410$ & 7845 & RAO $32 \mathrm{AC}$ & 5 & 175.3 & 24.84 & 6.5 & $\mathrm{O}$ (fast relative motion), not in FG-67 \\
\hline $02308+5533$ & 11696 & RAO $42 \mathrm{BE}$ & 1 & 207.0 & 15.677 & 5.11 & O (2MASS position, high crowding) \\
\hline $02355+5948$ & 12062 & $\mathrm{RAO} 43 \mathrm{AB}$ & 1 & 207.8 & 14.472 & 6.15 & O (2MASS position, high crowding) \\
\hline $02356+6106$ & 12067 & $\mathrm{RAO} 7 \mathrm{AB}$ & 1 & 169.0 & 5.71 & 5.1 & $\mathrm{P}(2 \mathrm{MASS}$ position $)$ \\
\hline $02370+2439$ & 12189 & $\mathrm{RAO} 8 \mathrm{BC}$ & 2 & 285.2 & 0.536 & 4.3 & $\mathrm{P}$ (fixed position, conf. with P200). $\mathrm{B}=\mathrm{HIP} 12184$ \\
\hline $02430+5812$ & 12685 & $\mathrm{RAO} 44 \mathrm{AB}$ & 1 & 211.1 & 21.670 & 4.08 & O? (color, high crowding) \\
\hline $02462+0536$ & 12925 & RAO $9 \mathrm{AB}$ & 3 & 252.8 & 1.91 & 4.4 & $\mathrm{P}$ (fixed position, low crowding) \\
\hline $02517+4559$ & 13336 & $\mathrm{RAO} 45 \mathrm{AC}$ & 1 & 81.8 & 15.195 & 6.30 & $\mathrm{O}$ (2MASS position) \\
\hline $03177+3838$ & 15329 & $\mathrm{RAO} 10 \mathrm{Ca}, \mathrm{Cb}$ & 3 & 223.4 & 1.560 & 4.0 & $\mathrm{P}$ (small separation, red color) \\
\hline $03305+2006$ & 16329 & RAO $11 \mathrm{Ba}, \mathrm{Bb}$ & 4 & 75.9 & 0.159 & 0.9 & $\mathrm{P}$ (spectroscopic binary), $\mathrm{B}=\mathrm{HD} 21663$ \\
\hline $03390+4232$ & 17022 & RAO $47 \mathrm{AB}$ & 1 & 142.9 & 1.741 & 6.2 & P (confirmed with P200) \\
\hline $03413+4554$ & 17217 & $\mathrm{RAO} 48 \mathrm{AC}$ & 1 & 269.0 & 4.83 & 6.4 & P (2MASS image) \\
\hline $03426+1718$ & 17336 & $\mathrm{RAO} 78 \mathrm{AB}$ & 1 & 269.7 & 10.388 & 6.44 & O (2MASS position) \\
\hline $03537+5316$ & 18218 & RAO 49 AC & 1 & 241.6 & 25.912 & 6.65 & O (2MASS position, high crowding) \\
\hline $04007+2023$ & 18719 & RAO $50 \mathrm{AB}$ & 1 & 308.4 & 5.905 & 6.35 & $\mathrm{O}$ (not found in 2MASS images) \\
\hline $04092+4010$ & 19389 & RAO $79 \mathrm{CD}$ & 2 & 294.3 & 9.584 & 3.04 & $\mathrm{O}$ (2MASS position) \\
\hline $04313+2008$ & 21099 & RAO $51 \mathrm{AB}$ & 1 & 310.3 & 18.800 & 6.32 & O (2MASS position) \\
\hline $04313+2008$ & 21099 & RAO $51 \mathrm{BC}$ & 1 & 177.4 & 0.917 & 0.26 & $\mathrm{O}$ ? (unrelated to A) \\
\hline $04363+5502$ & 21443 & $\mathrm{RAO} 35 \mathrm{AB}$ & 1 & 14.4 & 5.737 & 4.65 & P? (2MASS position \& color, confirmed with P200) \\
\hline $05017+2050$ & 23396 & RAO $52 \mathrm{AC}$ & 1 & 242.8 & 38.414 & 3.85 & O (2MASS position) \\
\hline $05096+2947$ & 24016 & RAO 53 BC & 1 & 106.2 & 26.153 & 6.58 & O (2MASS position), $\mathrm{B}=$ HIP 24005 \\
\hline $05247+6323$ & 25300 & $\mathrm{RAO} 36 \mathrm{AC}$ & 1 & 227.1 & 6.84 & 5.5 & P (2MASS image, conf. with P200) \\
\hline $05376+0607$ & 26444 & RAO $54 \mathrm{BC}$ & 1 & 34.9 & 4.526 & 5.79 & $\mathrm{O}$ (not in 2MASS image) \\
\hline $05376+0607$ & 26444 & RAO $54 \mathrm{BD}$ & 1 & 157.2 & 4.398 & 6.12 & $\mathrm{O}$ (not in 2 MASS image) \\
\hline $05444+4024$ & 27067 & RAO $55 \mathrm{AC}$ & 1 & 151.9 & 11.103 & 5.55 & O (2MASS position, high crowding) \\
\hline $05465+7437$ & 27246 & RAO $37 \mathrm{AC}$ & 1 & 68.5 & 10.85 & 3.5 & P (2MASS position, color) \\
\hline $06335+4822$ & 31267 & RAO $80 \mathrm{AC}$ & 1 & 221.9 & 4.97 & 5.3 & P (2MASS image, conf. with P200) \\
\hline $06562+4032$ & 33355 & RAO $56 \mathrm{AB}$ & 1 & 156.6 & 5.510 & 6.35 & P? (2MASS image, conf. with P200) \\
\hline $07043-0303$ & 34110 & RAO 57 AC & 1 & 10.6 & 15.713 & 6.64 & O (2MASS position) \\
\hline $07172+3306$ & 35265 & RAO $58 \mathrm{BC}$ & 1 & 126.6 & 17.637 & 5.45 & O (2MASS position, high crowding) \\
\hline $07475+7012$ & 38018 & RAO 59 AC & 1 & 118.8 & 15.476 & 5.87 & O (2MASS position) \\
\hline $08138+6306$ & 40298 & RAO $60 \mathrm{BC}$ & 2 & 37.6 & 13.074 & 3.48 & O (2MASS position) \\
\hline $08159+0227$ & 40479 & RAO $61 \mathrm{AB}$ & 1 & 170.6 & 31.245 & 6.42 & O (2MASS position) \\
\hline $08211+6527$ & 40918 & RAO $13 \mathrm{BC}$ & 1 & 245.6 & 2.79 & 3.2 & P (2MASS image), $\mathrm{B}=$ HIP 40882 \\
\hline $08258+1703$ & 41319 & RAO 62 BC & 2 & 310.0 & 16.798 & 4.19 & $\mathrm{O}$ (2MASS position) \\
\hline $08508+3504$ & 43426 & RAO 63 CD & 1 & 357.6 & 18.943 & 3.20 & O (2MASS position) \\
\hline $09505+0421$ & 48273 & $\mathrm{RAO} 90 \mathrm{Ba}, \mathrm{Bb}$ & 1 & 83.8 & 0.160 & 0.0 & $\mathrm{P}$ (close pair) \\
\hline $10079+6650$ & 49638 & RAO $15 \mathrm{AB}$ & 1 & 71.4 & 0.510 & 3.0 : & $\mathrm{P}$ (close pair, low crowding) \\
\hline $14094+1015$ & 69160 & RAO $16 \mathrm{AB}$ & 1 & 261.2 & 0.119 & 0.7 & $\mathrm{P}$ (SB2 resolved) \\
\hline $14113+5424$ & 69322 & RAO 17 BC & 2 & 145.9 & 0.577 & 1.7 & $\mathrm{P}$ (close pair, low crowding) \\
\hline $14418+2432$ & 71843 & RAO 64 BC & 2 & 61.9 & 17.271 & 2.02 & $\mathrm{O}$ (blue color) \\
\hline $15277+4253$ & 75676 & RAO $18 \mathrm{BC}$ & 1 & 100.2 & 0.408 & 1.5 & $\mathrm{P}($ close pair, small $\Delta m)$ \\
\hline $16147+3352$ & 79607 & RAO 65 EF & 1 & 238.2 & 32.267 & 3.74 & O (not found in 2MASS), $\mathrm{E}=$ HIP 79551 \\
\hline $16150+6040$ & 79629 & $\mathrm{RAO} 81 \mathrm{Ba}, \mathrm{Bb}$ & 1 & 42.1 & 0.139 & 0.0 & $\mathrm{P}($ close pair, small $\Delta m)$ \\
\hline $16400-0605$ & 81608 & RAO 82 BC & 1 & 148.9 & 27.160 & 3.89 & O (2MASS position) \\
\hline $17229-0223$ & 85042 & RAO $19 \mathrm{BC}$ & 1 & 86.3 & 0.752 & 0.0 & $\mathrm{P}($ close pair, small $\Delta m)$ \\
\hline $17422+3804$ & 86642 & $\mathrm{RAO} 20 \mathrm{AB}$ & 1 & 302.2 & 2.20 & 5.0 & P (confirmed with P200) \\
\hline $18123+0154$ & 89207 & RAO 66 AC & 2 & 71.9 & 17.237 & 6.81 & O? (2MASS position, crowded field) \\
\hline $18352+4135$ & 91120 & $\mathrm{RAO} 83 \mathrm{Ba}, \mathrm{Bb}$ & 1 & 336.1 & 0.136 & 0.8 & $\mathrm{P}($ close pair, small $\Delta m)$ \\
\hline $19145+3434$ & 94540 & RAO 84 AE & 1 & 72.2 & 2.900 & 4.50 & $\mathrm{O}$ ? (not confirmed in 2MASS image) \\
\hline $19158+3823$ & 94666 & RAO $85 \mathrm{AB}$ & 1 & 331.1 & 3.57 & 5.7 & P (2MASS image) \\
\hline $19188+1629$ & 94905 & RAO $67 \mathrm{AB}$ & 1 & 190.9 & 6.945 & 4.85 & $\mathrm{O}$ ? (very crowded field, small PM) \\
\hline
\end{tabular}


Table 5

(Continued)

\begin{tabular}{|c|c|c|c|c|c|c|c|}
\hline $\begin{array}{l}\text { WDS } \\
(2000)\end{array}$ & HIP1 & $\begin{array}{l}\text { Discoverer } \\
\text { Designation }\end{array}$ & $N$ & $\begin{array}{c}\theta \\
(\operatorname{deg})\end{array}$ & $\begin{array}{c}\rho \\
\left({ }^{\prime \prime}\right)\end{array}$ & $\begin{array}{c}\Delta m \\
(\mathrm{mag})\end{array}$ & Status, remarks \\
\hline $19234+2034$ & 95309 & RAO $68 \mathrm{AB}$ & 1 & 350.9 & 5.13 & 4.7 & P (2MASS position) \\
\hline $19359+5659$ & 96395 & RAO $87 \mathrm{AB}$ & 1 & 250.7 & 10.08 & 5.3 & P (2MASS position \& color) \\
\hline $19464+3344$ & 97222 & RAO $69 \mathrm{FK}$ & 1 & 318.3 & 16.402 & 6.21 & O (2MASS position) \\
\hline $20086+8507$ & 99232 & RAO $21 \mathrm{AC}$ & 2 & 65.3 & 1.32 & 5.5 & P? (small separation, low crowding) \\
\hline $20169+5017$ & 99965 & RAO $70 \mathrm{FL}$ & 1 & 264.2 & 15.597 & 4.66 & O (2MASS position, high crowding) \\
\hline $20312+5653$ & 101234 & RAO $22 \mathrm{AB}$ & 1 & 195.8 & 0.175 & 0.0 & P (acceleration binary, conf. with P200) \\
\hline $20333+3323$ & 101430 & RAO $71 \mathrm{AE}$ & 1 & 224.7 & 12.279 & 6.18 & O (2MASS position, high crowding) \\
\hline $20408+1956$ & 102040 & $\mathrm{RAO} 23 \mathrm{Ca}, \mathrm{Cb}$ & 1 & 59.4 & 0.266 & 0.0 & $\mathrm{P}$ (close and bright) \\
\hline $20408+1956$ & 102040 & RAO $23 \mathrm{CD}$ & 1 & 303.0 & 20.326 & 3.84 & $\mathrm{O}$ (not found in 2MASS, high crowding) \\
\hline $20408+1956$ & 102040 & RAO $23 \mathrm{CE}$ & 1 & 327.2 & 29.350 & 4.37 & $\mathrm{O}$ (not found in 2MASS, high crowding) \\
\hline $20577+2624$ & 103455 & RAO $24 \mathrm{AB}$ & 2 & 102.8 & 0.610 & 4.9 & P (acceleration binary, conf. with P200) \\
\hline $21102+2045$ & 104514 & RAO $25 \mathrm{AB}$ & 2 & 209.1 & 3.39 & 4.6 & P (2MASS image, conf. with P200) \\
\hline $21585+0347$ & 108473 & RAO $73 \mathrm{AB}$ & 1 & 89.3 & 12.39 & 5.6 & P (2MASS position \& color) \\
\hline $22094+3508$ & 109361 & RAO $26 \mathrm{AB}$ & 1 & 351.0 & 0.368 & 2.5 & $\mathrm{P}$ (aceleration \& SB1) \\
\hline $22204+4625$ & 110291 & RAO $74 \mathrm{AB}$ & 1 & 122.9 & 14.980 & 4.52 & O (2MASS position, crowding) \\
\hline $22204+4625$ & 110291 & $\mathrm{RAO} 74 \mathrm{AC}$ & 1 & 232.1 & 30.572 & 3.82 & O (2MASS position, crowding) \\
\hline $22240+0612$ & 110574 & $\mathrm{RAO} 27 \mathrm{Aa}, \mathrm{Ab}$ & 1 & 327.0 & 0.092 & 0.0 & P (acceleration binary, conf. with P200) \\
\hline $22246+3926$ & 110626 & RAO $28 \mathrm{AB}$ & 1 & 315.8 & 4.37 & 6.0 & P (2MASS image) \\
\hline $22311+4509$ & 111148 & RAO $29 \mathrm{BC}$ & 1 & 215.7 & 3.9108 & 6.00 & $\mathrm{O}$ (not found in 2MASS, high crowding) \\
\hline $22311+4509$ & 111148 & RAO $29 \mathrm{BD}$ & 1 & 264.4 & 12.276 & 3.59 & O (2MASS position, crowding) \\
\hline $22524+0950$ & 112935 & RAO $30 \mathrm{Da}, \mathrm{Db}$ & 4 & 319.3 & 0.242 & 0.2 & $\mathrm{P}$ (close, fixed for $1 \mathrm{yr})$ \\
\hline $23108+4531$ & 114456 & RAO 89 CD & 1 & 22.3 & 20.587 & 2.63 & O (2MASS position) \\
\hline $23108+4531$ & 114456 & RAO $89 \mathrm{CE}$ & 1 & 290.6 & 15.330 & 3.03 & O (2MASS position) \\
\hline $23258+4521$ & 115655 & RAO 75 BC & 1 & 65.4 & 13.195 & 5.79 & O?(2MASS color) \\
\hline $23258+4521$ & 115655 & RAO $75 \mathrm{CD}$ & 1 & 279.8 & 0.612 & 1.01 & $\mathrm{O}$ (unrelated to $\mathrm{B}$ ) \\
\hline $23419-0559$ & 116906 & RAO $31 \mathrm{Ba}, \mathrm{Bb}$ & 3 & 69.6 & 0.519 & 0.1 & P (close \& bright, fixed for $1 \mathrm{yr}$ ) \\
\hline
\end{tabular}

separation, color, crowdedness of the field, and proper motion of the main target. We do not compute the formal probability of physical association based on relative astrometry because the motion can be distorted by subsystems, while chance projections with small and similar motions do happen sometimes (see e.g., Tokovinin \& Lépine 2012). Other factors involved in the classification are difficult to quantify in terms of probability. Most faint companions in Table 5 wider that $10^{\prime \prime}$ are optical with a large confidence; only three of them have an uncertain status. In some instances, the companions are not found in the catalog, but the 2MASS images in the $K_{s}$ band reveal their presence as blends. This is taken as confirmation, because the companion position matches (at least qualitatively) and because the detection of the blend in 2MASS indicates that the companion is brighter in $K_{s}$ than in $i^{\prime}$, indicating it is a cooler object below the detection threshold in the catalog.

Notes in the Appendix give additional information on the multiple systems appearing in Table 5, the status of the measured binary pairs ("P" for true physical binaries, "O" for chance optical alignments of unrelated stars), and the reasons for this classification. Follow-up observations in September 2013 with the PALM 3000 AO system at the $5 \mathrm{~m}$ Hale telescope at Palomar Observatory (Dekany et al. 2013) are mentioned where relevant, while their full results will be published in a forthcoming paper (L. C. Roberts et al., in preparation). Two of the systems with newly resolved binaries, HIP 2292B (= HIP 2350) and HIP 12189B (= HIP 12184), are also exoplanet hosts; a complete discussion of their properties are included in Roberts et al. (2014). Two RAO pairs were also measured by speckle interferometry at the SOAR $4.1 \mathrm{~m}$ telescope (Tokovinin et al. 2014).

\subsection{Binarity of Secondary Components}

Overall, there are 212 secondary components with separations above $10^{\prime \prime}$ that were observed with Robo-AO. Figure 6 illustrates different hierarchies found in this subsample. The wide binary is the root of the hierarchy (level 1); in 112 cases there are no subsystems. Another 100 wide binaries contain inner hierarchies of level 11 (subsystem in the primary component), level 12 (subsystem in the secondary) or both (that is, a $2+2$ quadruple). Here we silently ignore several subsystems (levels 111 etc.), and restrict the discussion to the hierarchical levels 1,11 , and 12 . As some subsystems are most likely not yet discovered, it is safe to say that at least half of those wide binaries are in fact triple or higher-order multiples.

Orbital periods of wide binaries are estimated roughly from their projected separation and denoted as $P^{*}$ to distinguish them from orbital solutions. Masses of binary components and the mass ratio $\left(q=\mathcal{M}_{2} / \mathcal{M}_{1}\right)$ are estimated from their absolute magnitudes, assuming that the stars obey standard relations for the main sequence. The detection limits of imaging are converted from the observed parameters $(\rho, \Delta m)$ into binary parameters $\left(P^{*}, q\right)$, and combined with the detection limits from spectroscopy and other techniques (more details in Tokovinin 2014).

There are 83 primary (level 11) and 39 secondary (level 12) subsystems in the surveyed objects (Figure 7); 19 new secondary subsystems were found, doubling their previously observed 


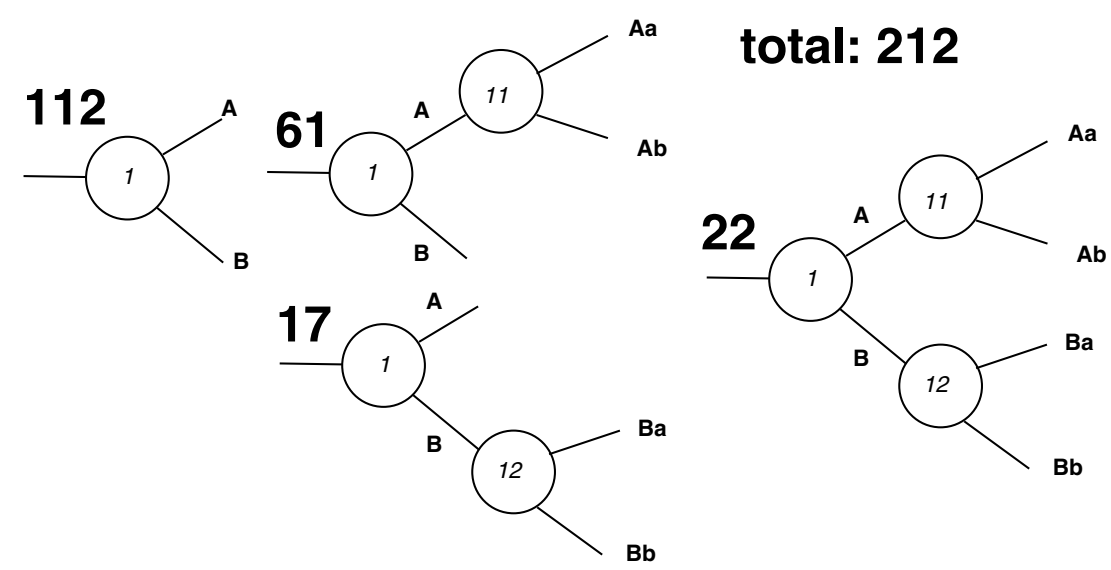

Figure 6. Observed numbers of different hierarchies in 212 wide binaries surveyed by Robo-AO.
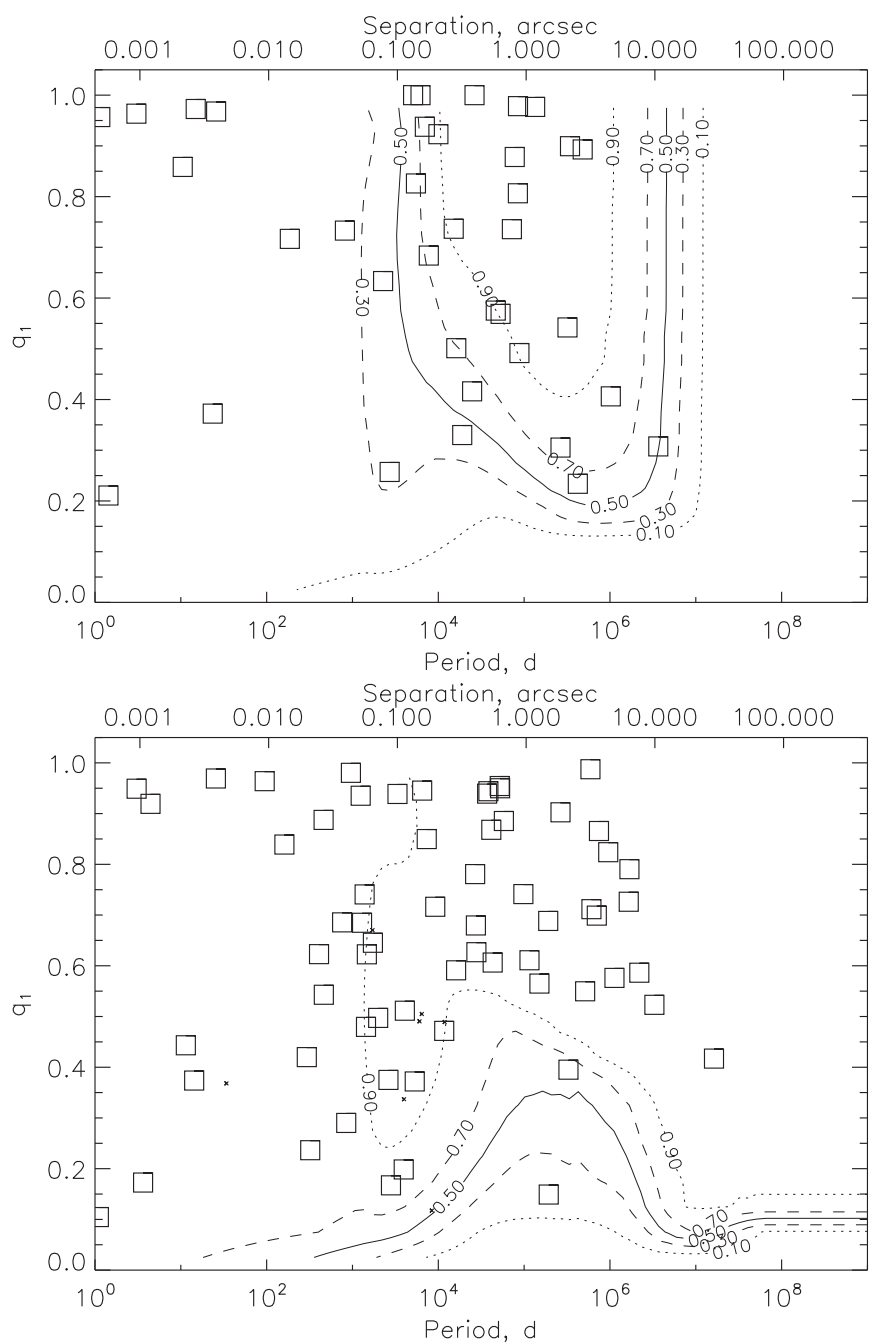

Figure 7. Distribution of 39 secondary subsystems (upper panel) and 83 primary subsystems (lower panel) of wide binaries in the $(P, q)$ plane. The contours indicate average detection probability. The angular separation on the upper axis corresponds to the distance of $50 \mathrm{pc}$.

fraction. At first glance, the primary subsystems still dominate, but remember that most of the primary targets (bright $\mathrm{F}$ and $\mathrm{G}$ dwarfs) were surveyed in radial velocity (RV), while only a few brighter secondaries have such RV coverage. The primaries also benefit from the Hipparcos discoveries of acceleration

subsystems (Makarov \& Kaplan 2005), which explains deeper detections at $P \sim 10^{3}$ d. At $P \sim 10^{6} \mathrm{~d}$ the situation is reversed in favor of secondaries, owing to their imagery with Robo-AO. At still longer periods (separation $\gtrsim 5^{\prime \prime}$ ), the companion census for primaries is complete because of 2MASS (Tokovinin 2011) and CPM (Tokovinin \& Lépine 2012) data, while for the secondaries the detections are restricted by the size of the Robo-AO field. However, this makes little difference because extremely wide subsystems are dynamically unstable.

It is convenient to express orbital periods by $x$, the logarithm of the period in days. The criterion of dynamical stability by Mardling \& Aarseth (2001) requires that the ratio of outer to inner periods in triple systems be greater than 4.7, depending on the eccentricity in the outer orbit. The dynamical truncation function $F(\Delta x)$ (where $\Delta x=x_{\text {out }}-x_{\text {in }}$ is the logarithm of the period ratio) is the probability of a given triple system being dynamically stable; it is modeled here as zero for $\Delta x<0.7$, one for $\Delta x>1.7$, and linear in between. Considering the large range in periods, the exact form of this function has little influence on the results.

In the subsample of 212 wide binaries, the average dynamical truncation is 0.95 at inner period $x_{\text {in }}=5$ and 0.5 at $x_{\text {in }}=6$, explaining the lack of wide subsystems at levels 11 and 12 . There is no need to look for binary secondaries with separations $>10^{\prime \prime}$ because those are intrinsically rare. The limitation imposed by the Robo-AO field of view is therefore not important for this work.

We select for analysis the period range $3.5 \leqslant x_{\text {in }} \leqslant 5$ where the dynamical truncation is not important and the detection of secondary subsystems with Robo-AO is relatively complete. This range corresponds to one decade in separation. We compare the frequency of primary and secondary subsystems in this range of periods among selected 212 wide binaries in Table 6 . The number of missed companions obviously depends on the distribution of the mass ratio, modeled as a power law 
$f(q) \propto q^{\beta}$. It is generally accepted that the power index $\beta$ for solar-type binaries is close to zero (uniform distribution) or slightly negative (Duchêne \& Kraus 2013), while low-mass binaries tend to have more equal components and $\beta \sim 1$.

The probability of detecting a subsystem averaged over period (in the selected interval) and over the mass ratio $q$ can be used to correct the raw companion frequency, if $\beta=0$. However, the data collected here indicate that in the secondary subsystems $\beta \sim 1$, requiring a smaller correction for missed binaries. Therefore, the estimated frequency of secondary subsystems with $3.5 \leqslant x_{\text {in }} \leqslant 5$ is $0.13 \pm 0.03$, assuming conservatively $\beta=1$. It is only slightly less than the frequency of primary (level 11) subsystems, which is less sensitive to the assumed $\beta$ and is somewhere between 0.13 and 0.15 (Table 6).

The frequency of subsystems found here is close to the frequency of solar-type binaries in the same period range. For the log-normal period distribution and the binary fraction of 0.46 derived by Raghavan et al. (2010), the fraction of companions with $3.5 \leqslant x_{\text {in }} \leqslant 5$ is 0.112 (a similar estimate is obtained for the full FG-67 sample). The frequency of subsystems in the components of wide binaries may be enhanced in comparison to the single stars, but only slightly.

The actual frequency of subsystems in secondaries may be even higher because of two additional factors. First, the methods used to identify wide secondaries introduce some selection against close binaries (Tokovinin \& Lépine 2012). Second, detection limits here may be over-estimated (see Section 3.4). When the assumed detection limits are deeper than in reality, the correction of the raw frequency for incomplete detection becomes smaller.

Quite surprisingly, the subsystems in primary and secondary are often found at the same time. Among the 39 secondary subsystems, 22 (more than half!) belong to $2+2$ quadruples (Figure 6). Considering that many secondary subsystems with short periods are still missed, the actual fraction of $2+2$ quadruples among 212 wide binaries should be more than $10 \%$. This surprising correlation likely has a root in stellar system formation processes and requires further study.

\subsection{Frequency of Tertiary Components}

In the second part of our survey we looked for distant companions to close binaries. In other words, the problem is "reversed" and we explore the hierarchy from inside-out. Similar studies were made by Tokovinin et al. (2006), Allen (2007), Rucinski et al. (2007), and others.

The second survey selects main targets that are close binaries with $P<100 \mathrm{yr}(x<4.56)$. Binaries with yet unknown periods, such as acceleration binaries from Hipparcos (Makarov \& Kaplan 2005) and/or spectroscopic binaries from Nordström et al. (2004), are included. We resolved three acceleration binaries (HIP 6653, 101234, 103455) with Robo-AO, while more were resolved by a targeted campaign with the NICI instrument (Tokovinin et al. 2012, 2013). However, as both acceleration and RV techniques involve a non-negligible false alarm probability, some of those presumed close binaries are in fact single stars.

For the purpose of statistical analysis, we restrict the sample to 241 inner binaries with known periods $P<100 \mathrm{yr}$. In the cases where even closer inner subsystems are present (e.g., the A-component of a visual binary is a spectroscopic pair), we select the inner-most (closest) pair and proceed outward. The 71 close binaries have additional outer (tertiary) companions; some of the tertiary components are themselves close binaries

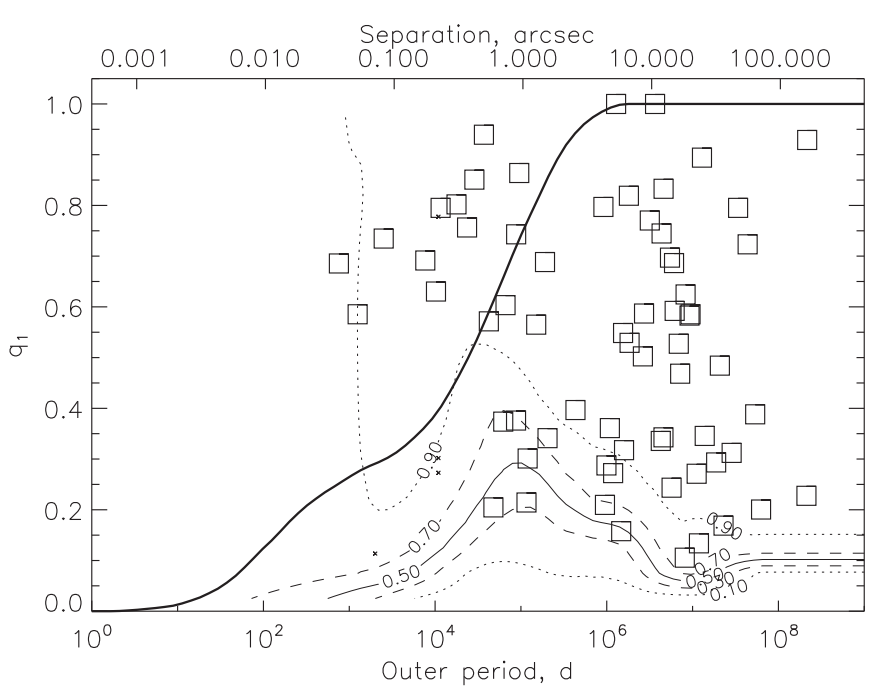

Figure 8. Periods and mass ratios of 71 tertiary components to 241 binaries with $P^{*}<100 \mathrm{yr}$ observed with Robo-AO. The contours indicate average detection probability. The full line over-plots the average dynamical truncation (not to be confused with $q$ ).

(in $2+2$ quadruples), and sometimes there are even more distant companions ( $3+1$ quadruples). The raw frequency of tertiaries is $29 \%$. When we restrict the sample to shorter inner periods, the fraction of triples increases, but the sample becomes smaller and the statistical errors increase.

Figure 8 presents tertiary components in the $\left(P_{\text {out }}, q_{1}\right)$ plane, where $q_{1}=\mathcal{M}_{3} / \mathcal{M}_{1}$ is the ratio of the masses between tertiary and primary components. The iso-detection curves go deeper than for the general sample (compare with Figure 7, lower panel) owing to the Robo-AO imaging. Many of tertiary components are found in the 2MASS catalog, but their colors alone were not sufficient to establish that they are physical, especially in crowded fields. The second-epoch imaging with Robo-AO helps here. A few uncertain cases (companions detected by Robo-AO in crowded fields without 2 nd epoch data) are not yet accepted as real.

The average dynamical truncation curve $F\left(x_{\text {out }}\right)$ is overplotted. It explains why tertiaries with short periods $x_{\text {out }} \leqslant 3$ are rare. Such tertiaries would be easily detectable by spectroscopy and their scarcity is genuine. As in Section 5.2, we evaluate the frequency of outer systems in the outer period range of $1.5 \mathrm{dex}$, $6 \leqslant x_{\text {out }} \leqslant 7.5$ - the range free from dynamical truncation and well covered by the detection techniques. It is safe to assume $\beta=0$ for wide components, in which case the average detection probability is 0.88 . The raw companion frequency of $34 / 241=$ $0.141 \pm 0.024$ is corrected to $0.161 \pm 0.028$. Owing to the deep detection limit, there is little difference between the raw and detection-corrected estimates. The frequency is significantly higher than the companion frequency to main targets in the same period range, 0.090 (again using the data of Raghavan et al. 2010).

It is premature to speculate about the meaning of this finding. In Figure 8 we note the "pile-up" of tertiary companions at $x_{\text {out }} \sim 7$ (separations on the order of $10^{\prime \prime}$ and projected separations on the order of $500 \mathrm{AU}$ ). If this feature is not a statistical fluctuation, it might imply some characteristic scale in the formation of solar-type multiple stars.

The overall raw frequency of tertiary components (with all periods) is only $29 \%$. If we assume that the intrinsic distribution of $x_{\text {out }}$ is Gaussian (Raghavan et al. 2010) and that their mass 
Table 7

New Orbital Elements

\begin{tabular}{|c|c|c|c|c|c|c|c|c|c|c|c|}
\hline WDS & HIP1 & Figure & $\begin{array}{c}P \\
(\mathrm{yr})\end{array}$ & $\begin{array}{c}a \\
\left({ }^{\prime \prime}\right)\end{array}$ & $\begin{array}{c}i \\
(\mathrm{deg})\end{array}$ & $\begin{array}{c}\Omega \\
(\operatorname{deg})\end{array}$ & $\begin{array}{c}T_{\circ} \\
(\mathrm{yr})\end{array}$ & $e$ & $\begin{array}{c}\omega \\
(\mathrm{deg})\end{array}$ & $\mathrm{Gr}$ & Ref. \\
\hline $01158+0947$ & 5898 & $9 a$ & $\begin{array}{r}162 . \\
\pm 13 \text {. }\end{array}$ & $\begin{array}{r}0.607 \\
\pm 0.035\end{array}$ & $\begin{array}{r}101.7 \\
\pm 1.0\end{array}$ & $\begin{array}{l}151.2 \\
\pm 2.1\end{array}$ & $\begin{array}{c}2101 \\
\pm 13 .\end{array}$ & $\begin{array}{r}0.447 \\
\pm 0.054\end{array}$ & $\begin{array}{r}262.8 \\
\pm 6.6\end{array}$ & 4 & $\bar{a}$ \\
\hline $01417-1119$ & 7916 & $9 b$ & $\begin{array}{r}601 \\
\pm 107\end{array}$ & $\begin{array}{r}2.42 \\
\pm 0.19\end{array}$ & $\begin{array}{r}82.6 \\
\pm 2.2\end{array}$ & $\begin{array}{r}267.98 \\
\pm 0.93\end{array}$ & $\begin{array}{r}2015.23 \\
\pm 0.87\end{array}$ & $\begin{array}{r}0.901 \\
\pm 0.030\end{array}$ & $\begin{array}{r}348.3 \\
\pm 9.7\end{array}$ & 4 & $\mathrm{~b}$ \\
\hline $02371-1112$ & 12204 & $9 \mathrm{c}$ & $\begin{array}{r}351 \\
\pm 271\end{array}$ & $\begin{array}{r}1.01 \\
\pm 0.34\end{array}$ & $\begin{array}{r}64.4 \\
\pm 3.4\end{array}$ & $\begin{array}{c}277 \\
\pm 12\end{array}$ & $\begin{array}{c}1902 . \\
\pm 15 .\end{array}$ & $\begin{array}{r}0.508 \\
\pm 0.044\end{array}$ & $\begin{array}{c}309 \\
\pm 43\end{array}$ & 5 & $\mathrm{c}$ \\
\hline $03140+0044$ & 15058 & $9 d$ & $\begin{array}{c}753 \\
\pm 84\end{array}$ & $\begin{array}{r}1.32 \\
\pm 0.13\end{array}$ & $\begin{array}{r}132.5 \\
\pm 2.8\end{array}$ & $\begin{array}{r}123.7 \\
\pm 7.8\end{array}$ & $\begin{array}{r}1883 \\
\pm 27\end{array}$ & $\begin{array}{r}0.281 \\
\pm 0.020\end{array}$ & $\begin{array}{c}254 \\
\pm 30\end{array}$ & 4 & $\mathrm{~d}$ \\
\hline $03413+4554$ & 17217 & $10 \mathrm{a}$ & $\begin{array}{r}46.2 \\
\pm 1.1\end{array}$ & $\begin{array}{r}0.27 \\
\pm 0.13\end{array}$ & $\begin{array}{r}90.3 \\
\pm 3.6\end{array}$ & $\begin{array}{r}269.9 \\
\pm 4.5\end{array}$ & $\begin{array}{r}1974.8 \\
\pm 1.7\end{array}$ & $\begin{array}{r}0.79 \\
\pm 0.76\end{array}$ & $\begin{array}{r}50.1 \\
\pm 9.0\end{array}$ & 4 & $\ldots$ \\
\hline $03496-0220$ & 17895 & $10 \mathrm{~b}$ & $\begin{array}{r}54 . \\
\pm 29\end{array}$ & $\begin{array}{r}0.49 \\
\pm 0.12\end{array}$ & $\begin{array}{r}114.5 \\
\pm 1.4\end{array}$ & $\begin{array}{c}234 \\
\pm 23\end{array}$ & $\begin{array}{r}1992.8 \\
\pm 3.4\end{array}$ & $\begin{array}{r}0.750 \\
\pm 0.012\end{array}$ & $\begin{array}{c}105 \\
\pm 12\end{array}$ & 4 & $\ldots$ \\
\hline $04163+0710$ & 19911 & $10 \mathrm{c}$ & $\begin{array}{r}12.38 \\
\pm 0.03\end{array}$ & $\begin{array}{r}0.2016 \\
\pm 0.0066\end{array}$ & $\begin{array}{r}69.99 \\
\pm 0.75\end{array}$ & $\begin{array}{r}144.8 \\
\pm 1.5\end{array}$ & $\begin{array}{r}2004.40 \\
\pm 0.04\end{array}$ & $\begin{array}{r}0.1677 \\
\pm 0.0043\end{array}$ & $\begin{array}{r}65.6 \\
\pm 1.5\end{array}$ & 3 & $\ldots$ \\
\hline $04422+3731$ & 21878 & $10 \mathrm{~d}$ & $\begin{array}{r}564 \\
\pm 119\end{array}$ & $\begin{array}{r}1.51 \\
\pm 0.22\end{array}$ & $\begin{array}{r}141.2 \\
\pm 6.0\end{array}$ & $\begin{array}{r}209.1 \\
\pm 7.6\end{array}$ & $\begin{array}{r}2024.9 \\
\pm 8.0\end{array}$ & $\begin{array}{r}0.4531 \\
\pm 0.0071\end{array}$ & $\begin{array}{c}262 . \\
\pm 11 .\end{array}$ & 4 & $\mathrm{e}$ \\
\hline $07043-0303$ & 34110 & $11 \mathrm{a}$ & $\begin{array}{r}46.88 \\
\pm 0.50\end{array}$ & $\begin{array}{r}0.273 \\
\pm 0.014\end{array}$ & $\begin{array}{r}99.2 \\
\pm 2.2\end{array}$ & $\begin{array}{r}99.4 \\
\pm 1.1\end{array}$ & $\begin{array}{r}1968.59 \\
\pm 0.76\end{array}$ & $\begin{array}{r}0.635 \\
\pm 0.037\end{array}$ & $\begin{array}{r}33.1 \\
\pm 5.9\end{array}$ & 3 & $\mathrm{f}$ \\
\hline $07092+1903$ & 34524 & $11 \mathrm{~b}$ & $\begin{array}{l}27.60 \\
\pm 1.1\end{array}$ & $\begin{array}{r}0.179 \\
\pm 0.013\end{array}$ & $\begin{array}{r}103.3 \\
\pm 1.9\end{array}$ & $\begin{array}{r}355.6 \\
\pm 1.7\end{array}$ & $\begin{array}{r}2010.81 \\
\pm 0.25\end{array}$ & $\begin{array}{l}0.94 \\
*\end{array}$ & $\begin{array}{r}244.5 \\
\pm 2.3\end{array}$ & 4 & $\ldots$ \\
\hline $08122+1739$ & 40167 & $11 \mathrm{c}$ & $\begin{array}{r}19.05 \\
\pm 0.46\end{array}$ & $\begin{array}{r}0.3728 \\
\pm 0.0068\end{array}$ & $\begin{array}{r}149.2 \\
\pm 7.2\end{array}$ & $\begin{array}{c}262 . \\
\pm 12 .\end{array}$ & $\begin{array}{r}1983.05 \\
\pm 0.32\end{array}$ & $\begin{array}{r}0.088 \\
\pm 0.013\end{array}$ & $\begin{array}{r}41 . \\
\pm 23 .\end{array}$ & 4 & $\mathrm{~g}$ \\
\hline $10387+0544$ & 52097 & $11 d$ & $\begin{array}{r}488.0 \\
\pm 3.6\end{array}$ & $\begin{array}{r}1.1962 \\
\pm 0.0051\end{array}$ & $\begin{array}{r}66 . \\
\pm 22 .\end{array}$ & $\begin{array}{r}159.89 \\
\pm 0.36\end{array}$ & $\begin{array}{c}1748 \\
\pm 14\end{array}$ & $\begin{array}{r}0.5967 \\
\pm 0.0018\end{array}$ & $\begin{array}{r}339.20 \\
\pm 0.29\end{array}$ & 4 & $\ldots$ \\
\hline $11293+3025$ & 56054 & $12 \mathrm{a}$ & $\begin{array}{r}254.9 \\
\pm 7.9\end{array}$ & $\begin{array}{r}1.18 \\
\pm 0.12\end{array}$ & $\begin{array}{c}117 \\
\pm 98\end{array}$ & $\begin{array}{c}246 . \\
\pm 20\end{array}$ & $\begin{array}{r}2075 . \\
\pm 61\end{array}$ & $\begin{array}{r}0.65 \\
\pm 0.12\end{array}$ & $\begin{array}{r}92 . \\
\pm 48 .\end{array}$ & 5 & $\ldots$ \\
\hline $13577+5200$ & 68193 & $12 b$ & $\begin{array}{l}205 \text {. } \\
\pm 20 \text {. }\end{array}$ & $\begin{array}{r}0.857 \\
\pm 0.028\end{array}$ & $\begin{array}{r}139.5 \\
\pm 9.2\end{array}$ & $\begin{array}{r}276.8 \\
\pm 5.5\end{array}$ & $\begin{array}{r}1911.6 \\
\pm 2.3\end{array}$ & $\begin{array}{r}0.717 \\
\pm 0.025\end{array}$ & $\begin{array}{c}330 . \\
\pm 11\end{array}$ & 3 & $\mathrm{~h}$ \\
\hline $15413+5959$ & 76837 & $12 \mathrm{c}$ & $\begin{array}{c}434 . \\
\pm 30\end{array}$ & $\begin{array}{r}1.013 \\
\pm 0.036\end{array}$ & $\begin{array}{r}74.4 \\
\pm 1.2\end{array}$ & $\begin{array}{r}211.83 \\
\pm 0.74\end{array}$ & $\begin{array}{r}1959.42 \\
\pm 0.95\end{array}$ & $\begin{array}{r}0.828 \\
\pm 0.016\end{array}$ & $\begin{array}{r}21.4 \\
\pm 2.2\end{array}$ & 4 & $\mathrm{i}$ \\
\hline $18154+5720$ & 89455 & $12 \mathrm{~d}$ & $\begin{array}{r}82 . \\
\pm 65\end{array}$ & $\begin{array}{r}0.40 \\
\pm 0.21\end{array}$ & $\begin{array}{r}62 . \\
\pm 14\end{array}$ & $\begin{array}{c}259 \\
\pm 19\end{array}$ & $\begin{array}{r}1997.8 \\
\pm 6.6\end{array}$ & $\begin{array}{r}0.36 \\
\pm 0.36\end{array}$ & $\begin{array}{l}313 \\
\pm 51\end{array}$ & 4 & $\ldots$ \\
\hline $20514-0538$ & 102945 & $13 a$ & $\begin{array}{r}199.8 \\
\pm 3.0\end{array}$ & $\begin{array}{r}0.844 \\
\pm 0.015\end{array}$ & $\begin{array}{r}65.13 \\
\pm 0.70\end{array}$ & $\begin{array}{r}173.36 \\
\pm 0.86\end{array}$ & $\begin{array}{r}1896.99 \\
\pm 0.81\end{array}$ & $\begin{array}{r}0.526 \\
\pm 0.014\end{array}$ & $\begin{array}{r}47.9 \\
\pm 2.4\end{array}$ & 2 & $\mathrm{j}$ \\
\hline $21041+0300$ & 103987 & $13 b$ & $\begin{array}{r}21.5 \\
\pm 6.6\end{array}$ & $\begin{array}{r}0.231 \\
\pm 0.041\end{array}$ & $\begin{array}{r}65.4 \\
\pm 6.2\end{array}$ & $\begin{array}{r}102.3 \\
\pm 6.6\end{array}$ & $\begin{array}{r}1985.0 \\
\pm 6.9\end{array}$ & $\begin{array}{r}0.24 \\
\pm 0.21\end{array}$ & $\begin{array}{r}25 \\
\pm 18\end{array}$ & 3 & $\ldots$ \\
\hline $21243+3740$ & 105676 & $13 c$ & $\begin{array}{r}17.8 \\
\pm 1.9\end{array}$ & $\begin{array}{r}0.167 \\
\pm 0.015\end{array}$ & $\begin{array}{r}122.1 \\
\pm 4.8\end{array}$ & $\begin{array}{r}247.3 \\
\pm 7.8\end{array}$ & $\begin{array}{r}2014.6 \\
\pm 1.3\end{array}$ & $\begin{array}{r}0.31 \\
\pm 0.10\end{array}$ & $\begin{array}{r}285.6 \\
\pm 7.4\end{array}$ & 3 & $\ldots$ \\
\hline $22161-0705$ & 109951 & $13 d$ & $\begin{array}{r}44 . \\
\pm 24\end{array}$ & $\begin{array}{r}0.30 \\
\pm 0.16\end{array}$ & $\begin{array}{r}36 . \\
\pm 101\end{array}$ & $\begin{array}{c}160 . \\
\pm 62 .\end{array}$ & $\begin{array}{r}1989.0 \\
\pm 5.3\end{array}$ & $\begin{array}{r}0.55 \\
\pm 0.59\end{array}$ & $\begin{array}{r}126 . \\
\pm 104 .\end{array}$ & 4 & $\mathrm{k}$ \\
\hline
\end{tabular}

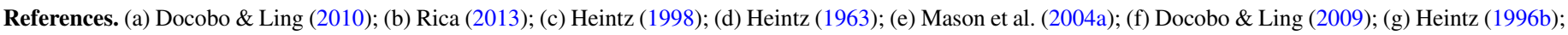
(h) Heintz (2001); (i) Heintz (1975); (j) Heintz (1998); (k) Cvetkovic (2011)

ratio $q_{1}$ is distributed uniformly, the companion frequency of this underlying distribution (before applying dynamical truncation and detection filters) is determined to be 0.54 ; i.e., slightly but not dramatically enhanced in comparison with 0.46 for all solar-type dwarfs. Most binaries with $P<100 \mathrm{yr}$ do not have outer tertiary companions; a similar conclusion was reached by Tokovinin et al. (2006) for spectroscopic binaries with periods of $\sim 10 \mathrm{~d}$ and longer. On the other hand, the closest binaries, with $P<3 \mathrm{~d}$, are found almost exclusively in triples.

\subsection{New and Updated Orbits}

For purposes of orbit determination, Robo-AO provides a unique collection of data. While there are certainly exceptions (Tokovinin 2012; Horch et al. 2012), the majority of interferometric data, whether with a filled or dilute aperture, have modest differential magnitude limits of about $\Delta m=3 \mathrm{mag}$. For many pairs of larger magnitude difference, the Robo-AO observation represent the first observation by a high-resolution technique, and given the decline in classical micrometry, the first observation of any kind in many decades. Given these factors, the number of first orbits as well as those with significant changes from the previous determinations is not surprising.

New orbits are presented in Table 7. In this table, the pairs are identified by their WDS and HIP1 numbers, followed by their orbital elements, giving the period $P$ in years, the semimajor axis $a$ in arcseconds, the inclination $i$ and longitude of the node $\Omega$, both in degrees, the epoch of the most recent periatron passage $T_{\circ}$ in Besselian years, the eccentricity $e$ and the longitude of periastron $\omega$ in degrees. Following this is the orbit grade (see Hartkopf \& Mason 2013b for details) as an evaluation of the orbit. For those pairs with previous orbit solutions we provide a reference to the previous "best" orbit. Figures 9-13 show the new orbital solutions, plotted with all published data in the WDS database. In each of these figures, micrometric observations are indicated by plus signs, high resolution measures by filled circles, Robo-AO observations as filled stars, and Hipparcos 

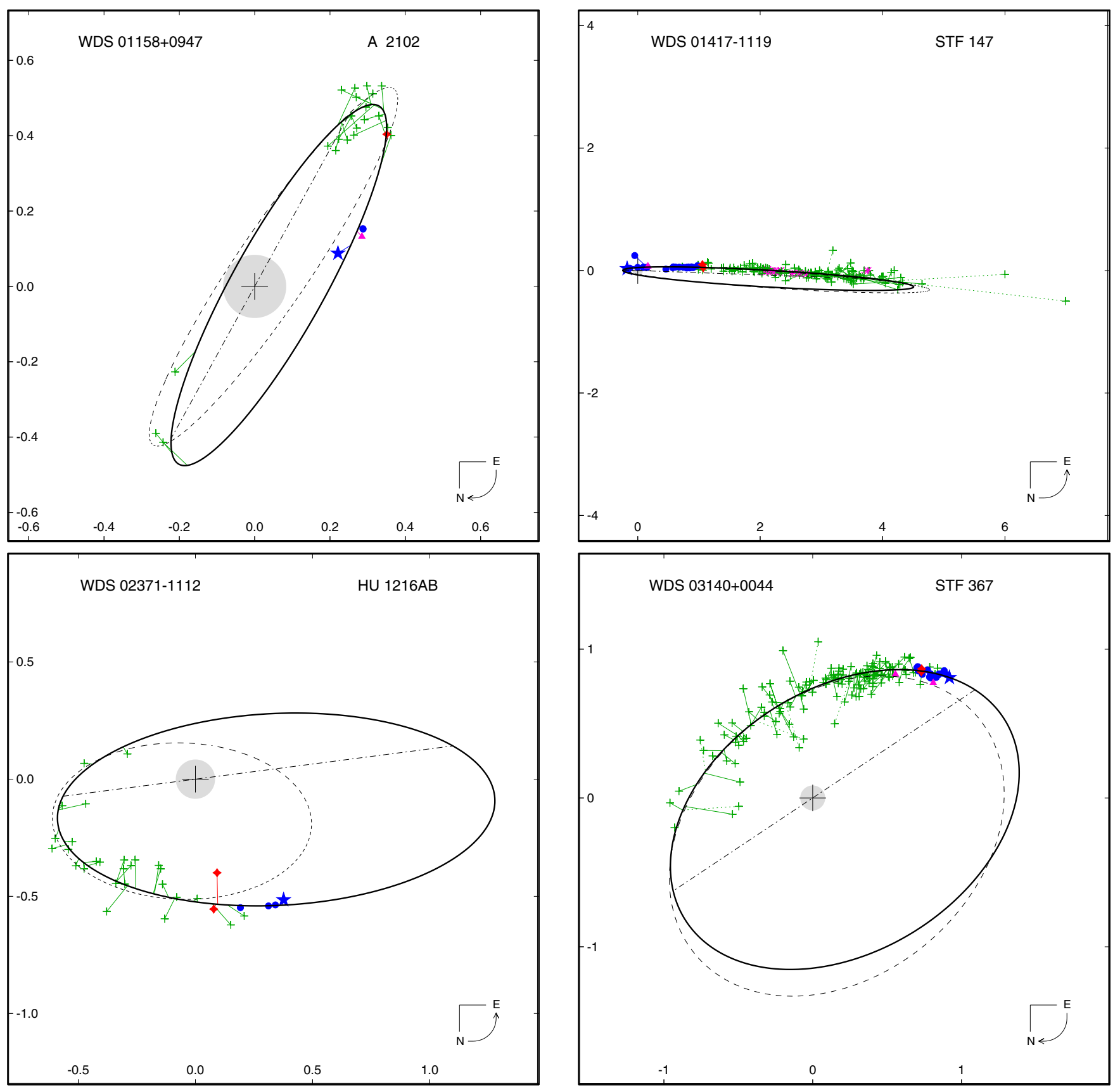

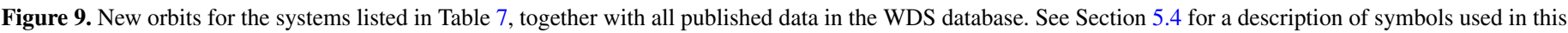

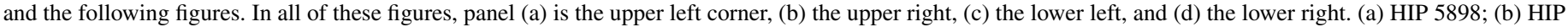
7916; (c) HIP 12204; (d) HIP 15058.

observations as filled diamonds. An $\mathrm{O}-\mathrm{C}$ line connects each measure to its predicted position along the new orbit (shown as a thick solid line). The shaded circle centered on the primary indicates the resolution limit of the P60. A dot-dashed line indicates the line of nodes, and a curved arrow in the lower right corner of each figure indicates the direction of orbital motion. Previous published orbits are shown as dashed ellipses; references to each of the published orbits are given in the notes to Table 7.

Orbital elements were determined with orbgrid10, the latest version of the venerable orbit reduction package of Hartkopf et al. (1989), which utilizes a three dimensional $(P, T$, and $e$ ) grid search of variable grid step sizes from three initial estimates.
It has been modified to include weighting methodologies determined in the production of the $5^{\text {th }}$ Catalog of Orbital Elements of Visual Binary Stars (Hartkopf et al. 2001). Some orbits use new speckle data from the SOAR telescope (Tokovinin et al. 2014).

The rest of this section gives notes on some pairs with new orbits.

HIP 12204 (=WDS J02371-1112= HU 1216AB) has only a preliminary orbit of grade 5 , hence the formal errors of the elements are very large.

HIP 17895 (=WDS J03496-0220 = YR 23): This is the first calculated orbit of this pair announced in Horch et al. (2002b) based on observations with the WIYN telescope. The 2004 

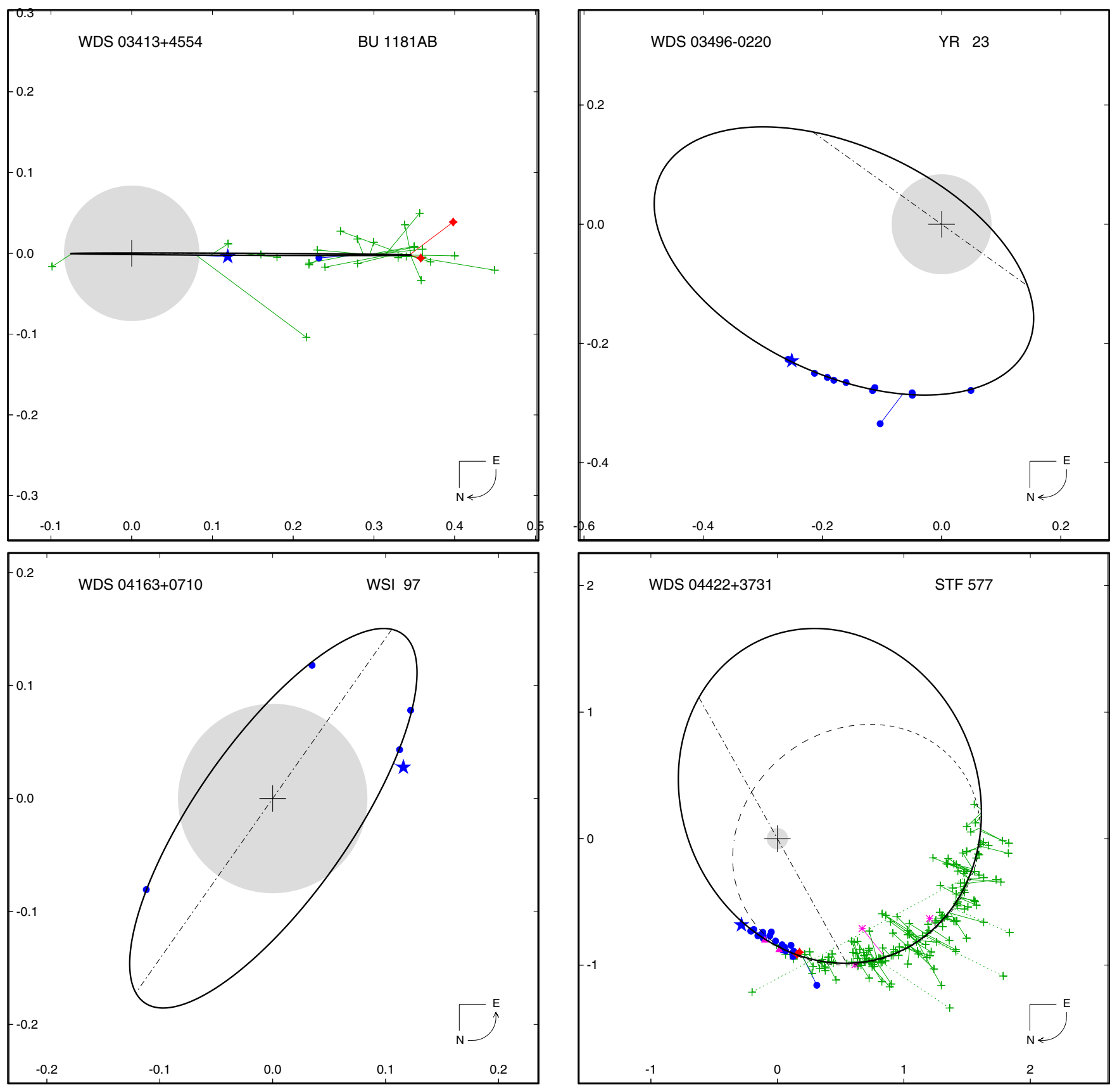

Figure 10. Same as Figure 9. (a) HIP 17217; (b) HIP 17895; (c) HIP 19911; (d) HIP 21878.

measure, obtained with the 26 inch telescope in Washington, D.C. (Mason et al. 2006), is off a bit, which is not surprising given the aperture.

HIP 34524 (=WDS J07092+1903 = CHR 216) was resolved by lunar occultations by Africano et al. (1975) and has several speckle measurements. Robo-AO finds it at nearly the same position as Hipparcos (the quadrant of our measure is established by the automatic data reduction co-added image), so the binary has made nearly one full revolution since 1991.25. It was not resolved at SOAR in 2009-2011 $\left(\rho<0^{\prime}\right.$.03), but measured in $2012.9,2013.05$, and 2014.06 (these data are used in the orbit calculation). The orbit is very eccentric, but measurements do not constrain the eccentricity, so it was fixed to $0.94-$ a provisional value that gives the expected mass sum of $1.8 \mathcal{M}_{\odot}$. The orbit should be followed by spectroscopy. Unfortunately, the periastron passage in 2010 was missed, we have to wait for the next one in 2038.

HIP 40167 (=WDS J08122+1739 = HUT 1Ca,Cb): This is the first calculated orbit of this pair. The existence of the $\mathrm{Ca}, \mathrm{Cb}$ pair was inferred and the first astrometric orbit calculated by Heintz (1996b).

HIP 103987 (=WDS J21041+0300=WSI 6): This is the first calculated orbit of this pair first split by Mason et al. (2001a) with the Struve $2.1 \mathrm{~m}$ telescope in an investigation of Hipparcos acceleration (G-type solution) pairs.

HIP 105676 (=WDS J21243+3740 = WSI 7): Like HIP 103987 above, this is the first calculated orbit of this pair also first resolved with the same telescope on the same project. 


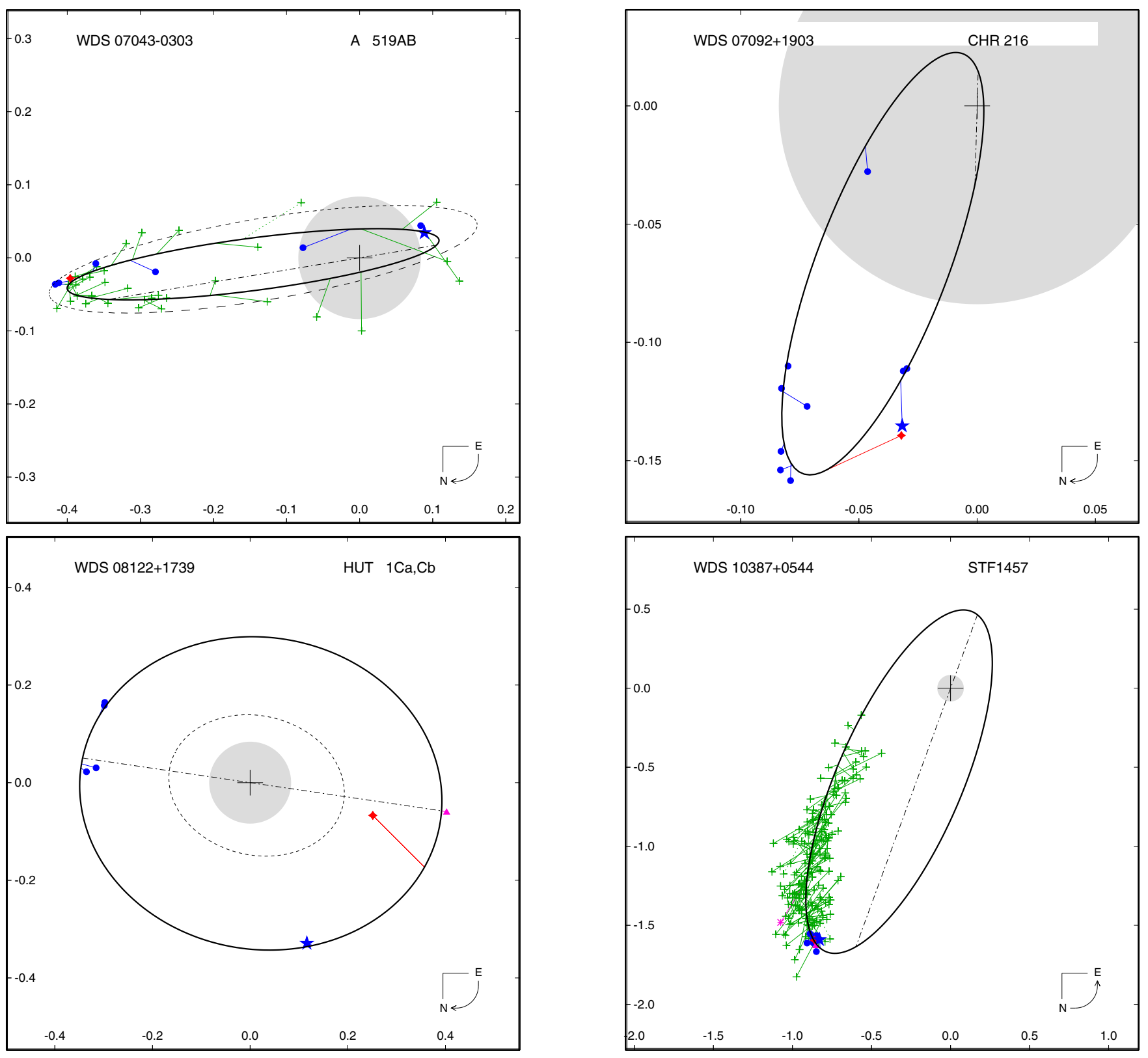

Figure 11. Same as Figure 9. (a) HIP 34110; (b) HIP 34524; (c) HIP 40167; (d) HIP 52097.

\section{SUMMARY}

A survey of high-resolution imaging with Robo-AO has advanced our knowledge of the multiplicity of solar-type stars in several ways.

The binarity of the wide secondary components turned out to be comparable to the frequency of subsystems in the primary components. This overturns the established paradigm about multiple-star architecture, where subsystems were thought to be preferentially associated with the primary, most massive star in the system. This traditional view can be traced to the previous technological restrictions allowing to study only bright stars by spectroscopy or high angular resolution, and matched the idea that multiple stars experience chaotic dynamical interactions where the least massive components are ejected onto distant orbits or leave the system altogether. This new paradigm of dynamical evolution of multiple star systems, where wide secondary components retain companions at the same frequency as the primary, will require more exploration to determine how such systems develop.

Quite unexpectedly, we discovered that subsystems in the primary and secondary components of wide binaries are often present simultaneously, i.e., these systems are in fact $2+2$ quadruples. This new result means that $2+2$ quadruples are relatively frequent. Note that in the $25 \mathrm{pc}$ sample of Raghavan et al. (2010), 9 out of 11 quadruples have the $2+2$ architecture and only 2 are of the $3+1$ "planetary" configuration; this result, based in small-number statistics, is now supported by the much larger FG-67 sample studied with Robo-AO. The high frequency of $2+2$ quadruples challenges the paradigm of chaotic $\mathrm{N}$-body dynamics (which can produce such architecture only exceptionally) and calls for exploration of other formation scenarios for multiple stars (e.g., Whitworth 2001). 

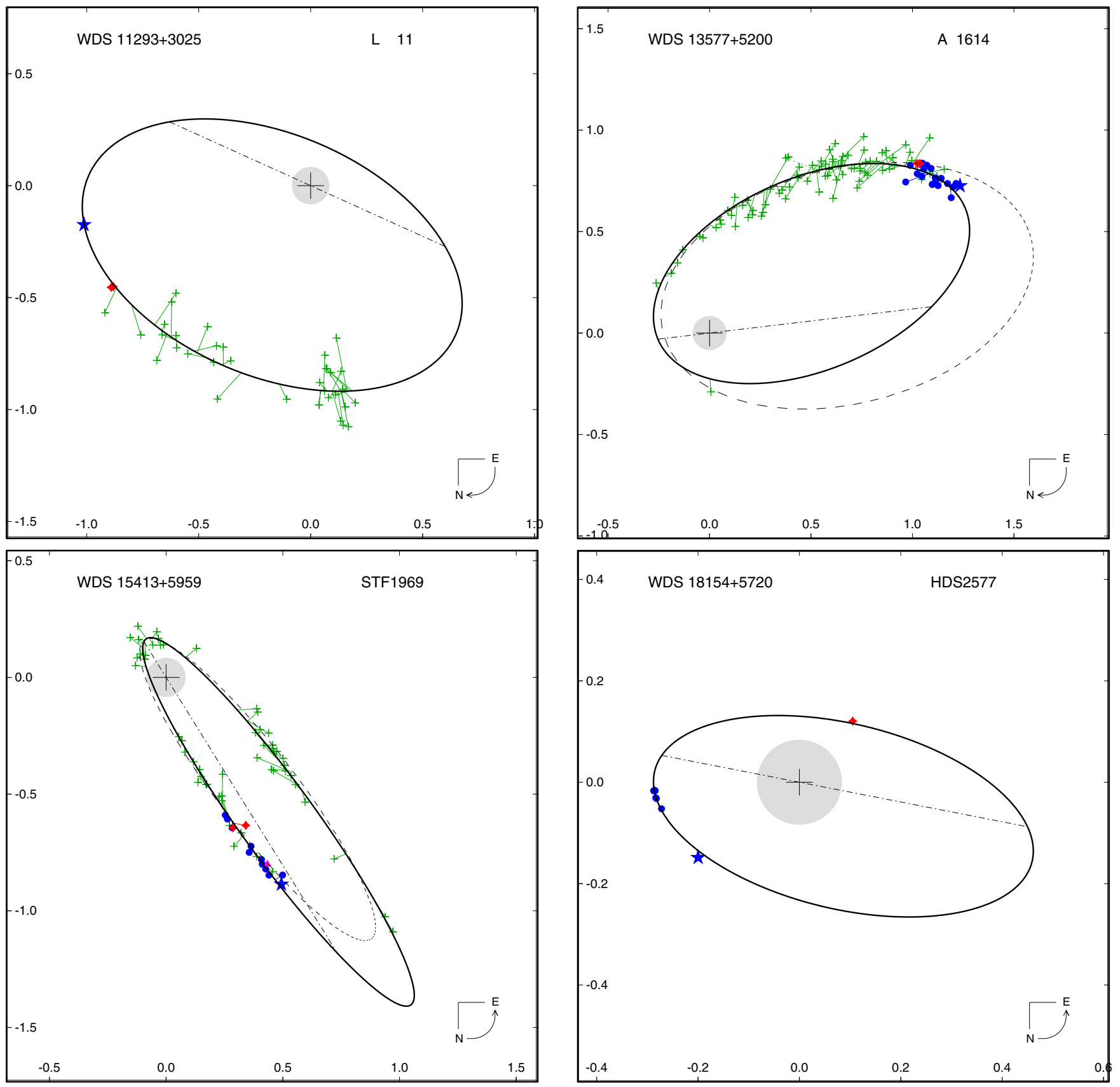

Figure 12. Same as Figure 9. (a) HIP 56054; (b) HIP 68193; (c) HIP 76837; (d) HIP 89455.

The high dynamic range of the Robo-AO imaging enabled discovery of 17 certain and 2 possible tertiary components to close binaries, converting them into triple or higher-order systems. Statistics of such triples reveal some interesting details, such as the prevalence of outer separations on the order of $500 \mathrm{AU}$. This result is not yet formally significant, but it highlights the potential of large samples. Historically small binary samples allowed only the first-order description of binary statistics by smooth functions with a few parameters (Duchêne \& Kraus 2013). Modern large and homogeneous samples begin to reveal new details in these distributions, such as twin binaries with identical masses (Lucy 2006), bimodal period distribution in Hyades (Griffin 2012), and, possibly, preferred separations of tertiary components seen here. These findings will advance our understanding of multiple-star formation.
The large data set that resulted from this survey enabled us to compute first visual orbits for 9 pairs and to update (sometimes dramatically) the existing orbits for 11 more. Accumulation and improvement of the visual-orbit data will lead to a better knowledge of stellar masses and to the statistical analysis of orbital elements. It will also enable dynamical study of visual binaries presenting special interest.

We acknowledge the input of the referee who read through this lengthy paper and gave us comments to improve it. The RoboAO system is supported by collaborating partner institutions, the California Institute of Technology and the Inter-University Centre for Astronomy and Astrophysics, and by the National Science Foundation under grant Nos. AST-0906060, AST0960343, and AST-1207891, by the Mount Cuba Astronomical 

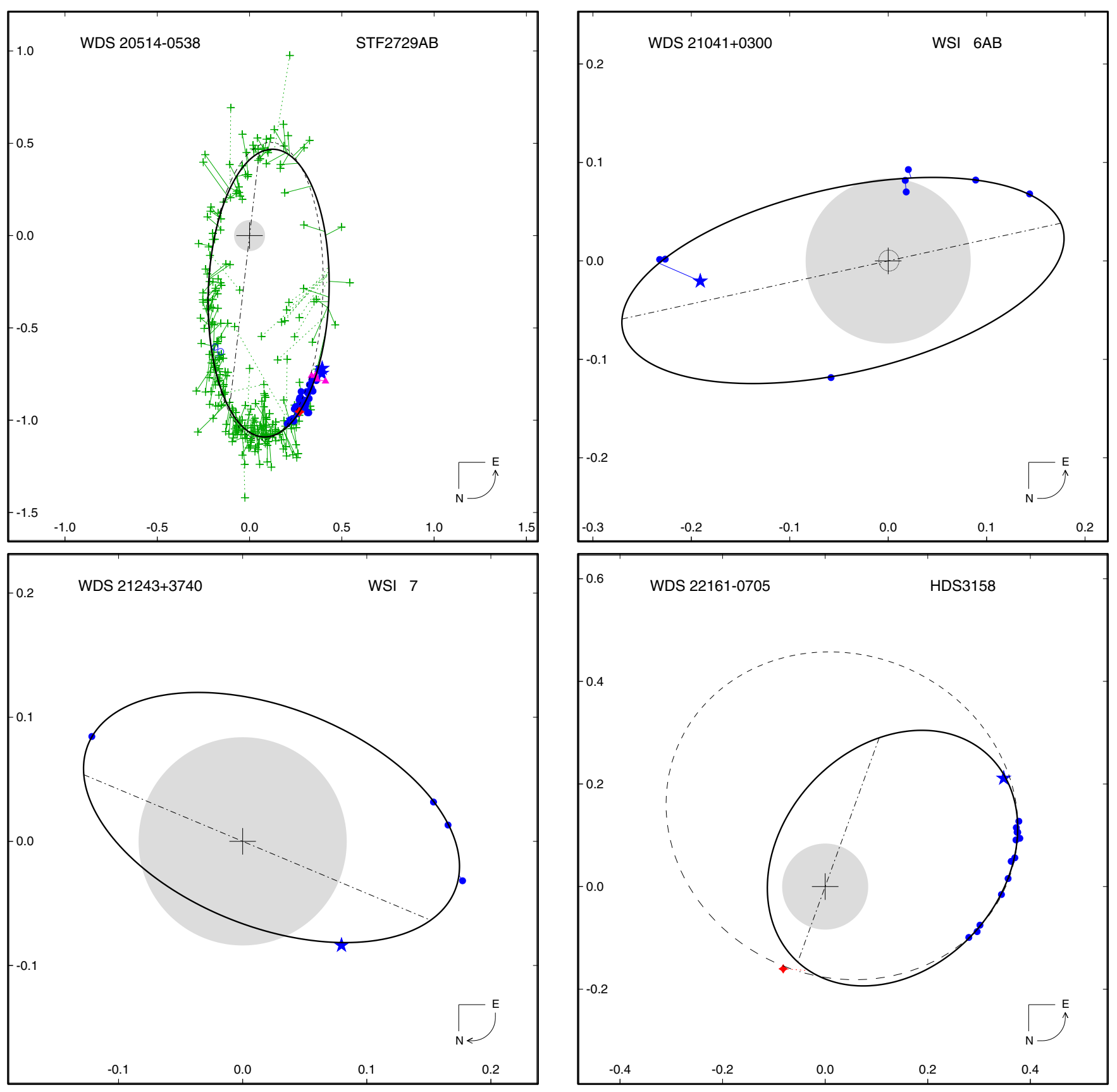

Figure 13. Same as Figure 9. (a) HIP 102945; (b) HIP 103987; (c) HIP 105676; (d) HIP 109951.

Foundation, and by a gift from Samuel Oschin. We are grateful to the Palomar Observatory staff for their ongoing support of Robo-AO on the P60, particularly S. Kunsman, M. Doyle, J. Henning, R. Walters, G. Van Idsinga, B. Baker, K. Dunscombe and D. Roderick.

A portion of the research in this paper was carried out at the Jet Propulsion Laboratory, California Institute of Technology, under a contract with the National Aeronautics and Space Administration.

C.B. acknowledges support from the Alfred P. Sloan Foundation.

This work used the SIMBAD service operated by Centre des Données Stellaires (Strasbourg, France), bibliographic ref- erences from the Astrophysics Data System maintained by SAO/NASA, data products of the Two Micron All-Sky Survey (2MASS), the Washington Double Star Catalog maintained at USNO.

Facility: PO:1.5m (Robo-AO)

\section{APPENDIX}

\section{NOTES ON NEW BINARY STAR SYSTEMS}

HIP 2292B = HIP $2350=$ RAO $1 \mathrm{BC}$ is resolved at 0'.5, with several Robo-AO measures and a confirmation with the Palomar 200 inch $(5 \mathrm{~m})$ telescope (P200). It remained fixed during $1 \mathrm{yr}$, despite the proper motion (PM) of $0.25 \mathrm{yr}^{-1}$. This star also hosts 
an exoplanet system with $P=3.44 \mathrm{~d}$. The common-propermotion (CPM) component A is at $839^{\prime \prime}$ from B. We discuss this multiple system in a separate paper.

HIP 2717B $=\mathrm{BD}+61119(V=8.98, \mathrm{~F} 8 \mathrm{~V}, \mathrm{X}$-ray source $)$ has two new companions at $\sim 3^{\prime \prime}$, designated as RAO $2 \mathrm{BC}$ and RAO 2BD. We see the brighter companion $\mathrm{C}$ in the 2MASS image at a similar position, although it is not listed as a separate point source in 2MASS. On the other hand, the fainter star D is likely optical because the field is crowded and because a trapeziumlike configuration of $\mathrm{BC}$ and $\mathrm{BD}$ would be dynamically unstable. The CPM nature of AB (Tokovinin \& Lépine 2012) is confirmed by the data from 2MASS and UCAC (Zacharias et al. 2013).

HIP 3214B ( $V=14.87)$ is resolved at 1 1".6. The new pair is considered physical because the companion is bright and because it remained fixed during $1 \mathrm{yr}$. The 2MASS image is elongated in the E-W direction.

HIP 3540 AB is LDS 836 at 55."4 separation. We resolved B into a new 1 1.5 pair RAO 4BC, confirmed with the P200. The component A is a spectroscopic binary (SB) with $P=11.4 \mathrm{~d}$ (Griffin 2002). The system is thus a $2+2$ quadruple.

HIP 3795 is another new quadruple system. The AB pair BU 495 has an orbit with $P=413$ yr. The CPM component $\mathrm{C}\left(V=10.83^{m}\right)$ at $152^{\prime \prime}$ from AB turns out to be a new 0.'67 pair $\mathrm{RAO} 5 \mathrm{Ca}, \mathrm{Cb}$. We consider $\mathrm{Ca}, \mathrm{Cb}$ physical because the companion is bright, the separation is small, and the field is not crowded.

HIP $4016 \mathrm{~B}$ is accompanied by the $1^{\prime \prime} .7$ pair $\mathrm{CD}$, at a distance of $17^{\prime \prime}$ from $\mathrm{B}\left(\mathrm{AB}=\mathrm{DAL} 11\right.$ at $41^{\prime \prime}$ is physical). The field is crowded, so we consider the faint stars $\mathrm{C}$ and $\mathrm{D}$ to be unrelated to B. BC is at $\left(267.2,15^{\prime \prime} .66\right)$ in 2MASS.

HIP 4878 has faint stars B and C at 2".9 and 32". 2 distance, respectively (RAO 39). The optical nature of $\mathrm{C}$ can be established by its different position $\left(145^{\circ} .3,32^{\prime \prime} .4\right)$ and its color in 2MASS. AB was remeasured with the P200 one year later and found at a slightly different position, so it is likely optical as well, although the field is not crowded.

HIP 5276 is an SB with $P=49.5$ d (Gorynya \& Tokovinin 2014), accompanied by the newly resolved tertiary component RAO 40 at 6.'2. The physical nature of the tertiary is confirmed by the 2MASS position and by observations with P200.

HIP 5313 is another triple system: $\mathrm{Aa}, \mathrm{Ab}$ is an acceleration and spectroscopic binary with a distant physical component B (RAO 41) at 26."2, confirmed by its position and color in 2MASS.

HIP 6653 has a new companion at 1".8 (RAO 6). The star is on the Keck exoplanet program, its radial-velocity (RV) trend (D. Fischer, private communication, 2012) could be caused by the companion. The companion B is red, the field has low crowding, so the physical nature of $\mathrm{AB}$ is likely.

HIP 7845 was measured as a test target, it is not in the FG-67 sample. The faint companion C at $24^{\prime \prime}$ moved substantially during a year, hence it is optical.

HIP 11696 is located in a crowded region of the sky. We targeted the component B and found a faint star at $15^{\prime \prime} .7$ from it (RAO 42BE), considered optical (just as the other components $\mathrm{C}$ and D listed in the WDS) because of the high crowding, non-hierarchical configuration, and the 2MASS position of BE.

HIP 12062 has an optical companion RAO 43 at 14".8. The pair has a different position in 2MASS and the region is crowded.

HIP 12067 has a new 5".7 companion B (RAO 7), confirmed by the 2MASS position, in addition to the inner acceleration and spectroscopic binary with yet undetermined period.
HIP 12189 and HIP 12184 form a 38" physical pair STFA 5 where each component is, in turn, a close binary: A is SB $(P=1.1 \mathrm{~d}), \mathrm{B}$ is resolved here into a $0^{\prime \prime} .53$ pair RAO 8, confirmed next year with Robo-AO and P200. Moreover, an exoplanet with $P=335 \mathrm{~d}$ around $\mathrm{B}$ was announced. We discuss this multiple system in a separate paper.

HIP 12685 has a companion at 21".7 (RAO 44) that is also present in 2MASS at approximately the same position. However, the PM is small, the field is crowded, and the color of B does not match a dwarf at the same distance as A, so we consider B optical.

HIP 12925 has a new companion at 1".9, measured several times with Robo-AO and confirmed with the P200. This is a young multiple system: the radial velocity (RV) of A is variable (Nordström et al. 2004), there is a CPM companion at 494" (Tokovinin \& Lépine 2012), and HIP 12862 at 54' is co-moving.

HIP 13336 contains the visual binary A 281AB and a companion RAO $45 \mathrm{AC}$ at $15^{\prime \prime}$, considered optical because it is found at a different position in 2MASS.

HIP 15329 contains the AB pair (STF 53, $P=113 \mathrm{yr}$ ) and a CPM companion $\mathrm{C}(V=13.24)$ at $107^{\prime \prime}$ found by (Tokovinin \& Lépine 2012). Here $\mathrm{C}$ is resolved at 1".6 (RAO $10 \mathrm{Ca}, \mathrm{Cb})$, revealing this system as a $2+2$ quadruple. We believe that $\mathrm{Ca}, \mathrm{Cb}$ is physical because of its small separation, moderate crowding, and the red color of $\mathrm{Cb}(\Delta i=4.0 \mathrm{mag}, \Delta z=2.8 \mathrm{mag})$.

HIP $16329 \mathrm{Ba}, \mathrm{Bb}=\mathrm{RAO} 11 \mathrm{Ba}, \mathrm{Bb}$ (also known as J 207) is a spectroscopic binary in the Hyades cluster with a period of about $30.74 \mathrm{yr}$ (Griffin 2012). Assuming the mass of the K7V component $\mathrm{Ba}$ to be $0.7 \mathcal{M}_{\odot}$, the spectroscopic orbit implies a minimum mass of $0.3 \mathcal{M}_{\odot}$ for $\mathrm{Bb}$ and a semi-major axis of 0 '.265. The pair $\mathrm{Ba}, \mathrm{Bb}$ was resolved by Robo-AO three times at 0 ' 16 with $\Delta i=0.87^{m}, \Delta r=0.97^{m}$, and $\Delta z=0.52^{m}$. This corresponds to a mass for Bb of $\sim 0.6 \mathcal{M}_{\odot}$. We can't help noting that $\mathrm{Bb}$ appears redder than $\mathrm{Ba}$, and that its lines were not detected in the spectrum; it could itself by a close binary or a fast rotator. This pair is a secondary component in the wide binary STF 399AB at 20" separation, also measured here (despite its WDS designation, AB was first resolved by W. Herschel in 1782, not by W. Struve). The primary component $A=v B 3$ is itself a close binary with astrometric acceleration (Makarov \& Kaplan 2005), although Griffin states that RV(A) is constant; it is not resolved by Robo-AO.

HIP 17022 has an astrometric orbit with $P=3.06 \mathrm{yr}$. This binary is too close to be resolved with Robo-AO, but we found another faint star at 1".7 (RAO 47) that is confirmed as physical with the P200.

HIP 17217 is a triple system with inner binary BU 1181 and a new component at 4". 8 (RAO 48AC) confirmed as physical by its presence in the $2 \mathrm{MASS}$ image.

HIP 17336 has a new companion at 10.'4 that is not found in the 2MASS point-source catalog, but is seen at $270^{\circ}$ and $12^{\prime \prime}$ from $\mathrm{A}$ in the $J$-band image. The difference in positions matches the reflex PM of $\mathrm{A}$, hence the new companion is optical.

HIP 18218 is the binary A $1293 \mathrm{AB}$. The new companion C at $26^{\prime \prime}$ is optical, as evidenced by its position in 2MASS and high crowding.

HIP 18719 is an SB in the Hyades (Griffin 2012) that is not resolved here. The faint star at $5^{\prime \prime} .9$ found here is optical because it is not seen in the 2MASS images.

HIP 19389 has a CPM companion C at 61" (Tokovinin \& Lépine 2012), targeted here. The pair CD is found in 2MASS, which shows that $\mathrm{D}$ is optical to $\mathrm{C}$, as the change in its position simply reflects the $\mathrm{PM}(\mathrm{C})$. 
HIP 21099 is a member of the Hyades. The faint 0.'9 pair BC seen at $19^{\prime \prime}$ from A is optical, as evidenced by its position in 2MASS.

HIP 21443 has a new companion B at 5".7, revealed as physical by its color and position in 2MASS and confirmed with the P200. However, the PM(A) is small and the region is crowded, so the status of the new companion is not certain. The main target is also an SB1 with a period of $2.06 \mathrm{~d}$ (Gorynya \& Tokovinin 2014).

HIP 23396 is the binary HU 445 that deviates from its orbit. The star C at $38^{\prime \prime}$ is classified as optical by comparing with its position in 2MASS.

HIP 24016 is a double-lined SB with a CPM companion B = HIP 24005 at $69^{\prime \prime}$. We targeted B and found a star C at 106.2, 26.2 from it in this crowded field. The star is at 103.9.1 and 27 ".32 in 2MASS, hence it is optical.

HIP 25300 is a system consisting of the $1^{\prime \prime} .1$ visual binary STF 677AB and a new tertiary component $C$ at 6."8, confirmed as physical by its presence in the 2MASS images. Observations with the P200 also confirmed C and resolved B into a close pair.

HIP 26444 is only triple - a single-lined SB coupled to the CPM component B at 229" distance (Tokovinin \& Lépine 2012). We targeted B and found two stars around it at $\sim 4^{\prime \prime}$. They are not present in the 2MASS $K_{S}$-band image and hence are considered optical.

HIP 27067 and HIP 27070 form the 22" binary STF 775. We measured another star $\mathrm{C}$ at $11^{\prime \prime}$ from $\mathrm{A}$ in this crowded field; $\mathrm{AC}$ is optical judging by its position in 2MASS.

HIP 27246 is a new triple. The $11.1 \mathrm{yr}$ spectroscopic and astrometric binary was recently resolved by Horch et al. (2012). The new component $\mathrm{C}$ at $10^{\prime \prime} \cdot 9$ is confirmed as physical by its position and color in 2MASS.

HIP 31267 is another triple system consisting of an SB and the newly found tertiary component $\mathrm{C}$, confirmed as physical by the 2MASS image and follow-up observations with the $\mathrm{P} 200$. The $53^{\prime \prime}$ pair UC $1450 \mathrm{AB}$ is not physical because the red color of the secondary places it well above the main sequence, while the small common PM is likely a chance coincidence.

HIP 33355 is an SB with a new companion at $5^{\prime \prime} .5$ that could be physical. The $K_{s}$-band image in 2MASS is extended toward a position angle of $90^{\circ}$ and the companion is confirmed with the P200. Yet, the small PM of the target makes it difficult to make a firm conclusion on the companion's status.

HIP 34110 is a triple system with a $5.1 \mathrm{~d}$ SB in the visual pair A 619, which deviates substantially from its orbit. The new component $\mathrm{C}$ at $15^{\prime \prime} .7$ is optical as shown the $2 \mathrm{MASS}$ data.

HIP 35265 has a wide CPM companion B at 927" (Tokovinin \& Lépine 2012), whose physical nature is not certain, but likely, given the substantial PM. We targeted B and measured RAO $58 \mathrm{BC}$ at 17 ".6. This pair has a different position in 2MASS, hence is optical.

HIP 38018 has an optical companion C at 118.7, 15."4, with a different position in 2MASS.

HIP 40298 has a CPM companion B at 244" (LDS 2564), which was targeted. The pair BC found here is optical.

HIP 40479 has an optical companion at 31", with different position in 2MASS.

HIP 40918 has a CPM companion B = HIP 40882 at 258" which is resolved here into the 2".8 pair RAO $13 \mathrm{BC}$ and confirmed by the 2MASS image. Considering that A itself is a long-period $\mathrm{SB}$, this is another $2+2$ quadruple discovered by this survey.
HIP 41319 has a CPM companion at 692", likely physical considering its large PM. It was targeted here, but the 16.'8 pair RAO 62BC is optical, as revealed by its different position in 2MASS.

HIP 43426 is a visual triple with two companions at 3.'6 and $49^{\prime \prime}$ from it. The distant component $\mathrm{C}$ was targeted. We measured RAO 63CD at 18.'9, but this pair is optical.

HIP 48273 is a double-lined SB with $P=3.955 \mathrm{~d}$ (Griffin \& Suchkov 2003) and has a CPM companion B at $1155^{\prime \prime}$ (Tokovinin \& Lépine 2012), considered physical because of the substantial common PM and matching colors. This secondary was resolved here at $0{ }^{\prime} 16$ (RAO 90Ba, Bb), converting the system into a quadruple. The pair $\mathrm{Ba}, \mathrm{Bb}$ was measured at $\mathrm{SOAR}$ on 2013.13 at similar position.

HIP 49638 is an astrometric binary with $P=4.2 \mathrm{yr}$ and an estimated semi-major axis of $0^{\prime} .05$. We detect the new companion RAO 15 at $0^{\prime \prime} .5$, converting this into a triple system.

HIP 69160 is a double-lined SB with $P=8.4 \mathrm{yr}$ (D. Latham, private communication, 2012) and expected semi-major axis of 0.09 . It is resolved here at 0.12 .

HIP 69322 has a CPM component B $(V=13.92)$ at $606^{\prime \prime}$ (Tokovinin \& Lépine 2012), which is resolved twice into a new 0.'58 pair RAO 17BC.

HIP 71843 has a CPM component B at $216^{\prime \prime}, V=14.9$. We measured a pair BC at $17^{\prime \prime} .3$ twice. Its position in the $2 \mathrm{MASS}$ is similar, but C is "bluer" than B, hence it could be a white dwarf, although more likely it is a background star, considering the small PM of the system.

HIP 75676 is a $40^{\prime \prime}$ physical binary KU 108 known since 1893. Both its components are SBs (D. Latham, private communication, 2012). In addition, we found the new 0.'4 pair BC, making this a quintuple system.

HIP 79607 is a known quintuple system. The component E (STF 2032AE, E = HIP 79551 = GJ 615.2C) is resolved here at O'.4 (but not for the first time: $E a, E b=Y S C ~ 152)$. Another star $\mathrm{F}$ at $32^{\prime \prime}$ from E (RAO 65) appears optical; it is not found in 2MASS.

HIP 79629 has a CPM component B at $107^{\prime \prime}, V=14.2$. It is resolved here into a 0.14 pair of equal stars, converting binary into a triple.

HIP 81608 has a CPM component B at 179". The $27^{\prime \prime}$ pair RAO $82 \mathrm{BC}$ is optical, it has a different position in 2MASS.

HIP 85042 has a CPM companion B at 49" according to Raghavan et al. (2010). The secondary is resolved here at $0^{\prime \prime} 75$ (RAO 19BC), while A itself is single.

HIP 86642 is a double-lined SB (D. Latham, private communication, 2012) with a newly discovered tertiary component at 2 ".2. The 2 MASS image does not resolve this pair, but it is confirmed with the P200, where the inner binary was also resolved.

HIP 89207 is a $2^{\prime \prime}$ binary AB (A 2260). Another star C found here at $17^{\prime \prime}$ is likely optical, as the field is very crowded.

HIP 91120 has a CPM companion B at $621^{\prime \prime}, V=11.14$. It is resolved here at 0 '. $14(\mathrm{RAO} 83 \mathrm{Ba}, \mathrm{Bb})$.

HIP 94540 is a visual triple system where the $1^{\prime \prime}$ pair BU $975 \mathrm{BC}$ is located at 33."6 from A. However, it was outside the Robo-AO field, and we measured only the known optical pair $\mathrm{AD}$ at $15^{\prime \prime} .9$ and the new companion $\mathrm{E}$ at 2.9 (RAO 84AE). The star E is not seen in the $K_{s}$-band 2MASS image and is presumed to be optical, until proven otherwise.

HIP 94666 is an SB for which we find a distant companion at $3^{\prime \prime} 6$, confirmed in the 2 MASS image. 
HIP 94905 is a double-lined SB with $P=5.38 \mathrm{~d}$. The companion RAO 67 at 6.9 has the same position in 2MASS. However, the field is extremely crowded and the PM(A) is small, so the new companion is considered to be likely optical.

HIP 95309 is similar to the previous case: we find a new 5 ". 1 companion to the SB in a very crowded field. However, the $\mathrm{PM}(\mathrm{A})$ is $0^{\prime} .2 \mathrm{yr}^{-1}$, so comparison with the 2 MASS position confirms the physical nature of RAO 68.

HIP 95769 is a spectroscopic and astrometric binary with $P=2.26$ yr. The companion B at 9'.9 is optical; it has a different position in 2MASS and the field is crowded.

HIP 96395 is an SB converted into a triple by establishing the physical nature of the $10^{\prime \prime}$ companion noted previously by Fuhrmann (2004). Its position in 2MASS is same as measured here, its color matches a main-sequence dwarf.

HIP 97222 is the binary STF 2576. It forms a quadruple or quintuple system with HIP 97295 at $792^{\prime \prime}$, which is the primary (Raghavan et al. 2010). The field is crowded and several optical components are listed in the WDS. We add to this list another optical pair RAO 69FK at 16."4.

HIP 99232 appears to be triple. It was resolved at 0.065 as WSI 109 (not confirmed yet by a second measure), while Robo-AO finds a faint companion C at $1^{\prime \prime} .9$, reobserved within a year. We consider RAO 21 AC physical (the field is not crowded), although a larger time base is needed for a solid confirmation because $\mathrm{PM}(\mathrm{A})$ is small.

HIP 99572 has a CPM companion $\mathrm{C}$ at $1021^{\prime \prime}(\mathrm{AC}=\mathrm{TDT}$ $2085)$ that was targeted by Robo-AO. The $18^{\prime \prime}$ pair RAO $88 \mathrm{CD}$ is optical (D is not found in 2MASS).

HIP 99965: we targeted the CPM companion $\mathrm{F}$ ( $V=13.5$, $\mathrm{AF}=\mathrm{GIC} 155$ ) at $106^{\prime \prime}$ and measured another star in the field; the pair RAO 70FL is optical, its position in 2MASS is very different.

HIP 101234 is an acceleration binary, resolved here for the first time into a 0.17 pair of equal stars RAO 22, and confirmed with the P200.

HIP 101430 appears to be a quadruple system. The outer $17^{\prime \prime}$ pair $\mathrm{AB}=\mathrm{HJ} 1535$ is composed of the spectroscopic and astrometric binary $\mathrm{Aa}, \mathrm{Ab}$ and a $0^{\prime \prime} .17$ pair $\mathrm{Ba}, \mathrm{Bb}$ tentatively seen in the pipeline RoboAO image, but not confirmed by speckle processing. This subsystem is not listed in Table 6 for that reason, but it was resolved with the P200 nevertheless. The $12^{\prime \prime}$ pair RAO 71AE is optical, with a different position in 2MASS in a crowded field.

HIP 102040: The CPM companion $\mathrm{C}$ at $125^{\prime \prime}$ from $\mathrm{A}(\mathrm{AC}=$ LDS 1045) was targeted and resolved at $0^{\prime} .27$ (RAO 23Ca,Cb). The two other wide companions D and E are optical; they are not recovered in 2MASS, while the field is crowded.

HIP 103455 is an acceleration binary resolved here at 0.6 (RAO 24) and confirmed later with the P200. Metchev \& Hillenbrand (2009) did not resolve this system, although its separation implies an orbital period of several decades.

HIP 103641 is a quintuple system that consists of two visual pairs COU 2431Aa,Ab (Aa is also a close SB) and HDS 2989Da,Db (HIP 103052) at 1132" from A. We targeted D and measured the close pair, as well as an optical star at 19 .7 from it (RAO 72DE), which has a different position in 2MASS. The WDS components $\mathrm{B}$ and $\mathrm{C}$ are also optical (crowded field).

HIP 104514 has variable RV according to Nordström et al. (2004). We found a new physical companion at 3..4 and measured it twice. The companion is confirmed in the 2MASS image and with the P200.
HIP 108473 is a 7.18 yr SB with a new physical companion RAO 73 at 12".4, confirmed by its position and color in 2MASS.

HIP 109361 is an acceleration binary with variable RV, resolved here at 0'37 (RAO 26).

HIP 110291 is astrometric and spectroscopic binary with $P=2.11$ yr. The two wide companions $\mathrm{B}$ and $\mathrm{C}$ measured in this crowded field are optical, as evidenced by their respective positions in 2MASS.

HIP 110574 is acceleration binary resolved here at $0{ }^{\prime \prime} 09$ and confirmed with the P200 and at SOAR. The CPM companion B at $171^{\prime \prime}(V=15.88)$ was not targeted, being too faint.

HIP 110626 is also an acceleration binary, but the new physical companion at 4".4 discovered by Robo-AO (and confirmed in the 2MASS image) is too distant to cause the acceleration. The system is therefore triple.

HIP 111148 has a CPM companion B $(V=13.74)$ at $62^{\prime \prime}$, $\mathrm{AB}=\mathrm{LEP} 108$. We targeted $\mathrm{B}$ and found two faint stars $\mathrm{C}$ and $\mathrm{D}$ at 3.'9 and 12.'3, respectively. Both companions are considered optical ( $\mathrm{C}$ is not seen in the 2MASS image, $\mathrm{D}$ has a different position of $268.3,14^{\prime \prime} .59$, and the field is crowded).

HIP 112935 is triple. Its CPM companion $\mathrm{D}(V=13.3$, $\mathrm{AB}=\operatorname{LDS} 6388)$ at $250^{\prime \prime}$ is resolved here at $0^{\prime \prime} .24$ several times at fixed position during one year, despite the fast PM of $0.5 \mathrm{yr}^{-1}$.

HIP 114456 has a CPM companion C at 50" (Raghavan et al. 2010) (the WDS pair HJ $1853 \mathrm{AB}$ is optical). We found only two optical stars around C, RAO 89CD and CE. They have different positions in 2MASS.

HIP 115655 has a CPM companion B = HD 20748 at 185". We measure a faint $\mathrm{CD}$ pair at $13^{\prime \prime}$ from $\mathrm{B}$. The color of $\mathrm{C}$ in 2MASS, $J-K=0.65$, suggests that it is optical, although $\mathrm{PM}(\mathrm{A})$ is small and the position of BC in 2MASS is close to its measured position.

HIP 116906 has a CPM companion B at 109'.5 (LDS 5112, $V=14.5)$, which is resolved here into a $0^{\prime \prime} .5$ pair $\mathrm{RAO} 31 \mathrm{Ba}, \mathrm{Bb}$ (three measurements within a year). The main star hosts an exoplanet with $P=572 \mathrm{~d}$.

HIP 118213 is an acceleration binary. We discover a tertiary companion at 4 .'8, confirmed by its repeated measurement within a year and seen in the 2MASS image. The new pair RAO 76 was also measured with the P200, where the inner binary was resolved as well.

HIP 118225 is an SB with $P=25.4 \mathrm{~d}$. We discovered another component at $5^{\prime \prime}$, confirmed by its repeated measurement and by the 2MASS image.

Table 8 summarizes the position measurements for the Robo$\mathrm{AO}$ and 2MASS data used to determine if components are physical or optical pairs in many of the newly resolved systems. Some of the Robo-AO pairs were not resolvable by 2MASS, so they are not included in the table. The table lists the binaries by HIP number, and the components that are being measured, followed by the measured angle (in degrees) and separation (in arcseconds) for the Robo-AO and 2MASS data, and the epoch of observation by 2 MASS. The column $\Delta$ gives the total displacement between the Robo-AO and 2MASS measurements. The next column $\mu \Delta t$ gives the displacement produced by proper motion, assuming $\Delta t=12 \mathrm{yr}$. The last two columns are the proper motion of the main target.

The 2MASS measurements at $\sim 5^{\prime \prime}$ separations are likely less accurate than its astrometry in general ( $\sim 70$ mas). The Robo-AO astrometry of wide pairs is accurate to $\sim 0^{\prime}$. 1 (Law et al. 2014b). The PM of companion candidates is unknown and comparable to the PM of some targets. Considering that the relative PM is 
Table 8

Comparison of Positions Measured by Robo-AO with 2MASS

\begin{tabular}{|c|c|c|c|c|c|c|c|c|c|c|}
\hline HIP1 & Comp & $\begin{array}{l}\theta_{\mathrm{RAO}} \\
(\mathrm{deg})\end{array}$ & $\begin{array}{c}\rho_{\mathrm{RAO}} \\
(\operatorname{arcsec})\end{array}$ & $\begin{array}{c}\text { 2MASS } \\
\text { Epoch }\end{array}$ & $\begin{array}{c}\theta_{2 \text { MASS }} \\
(\operatorname{deg})\end{array}$ & $\begin{array}{l}\rho_{2 \mathrm{MASS}} \\
(\operatorname{arcsec})\end{array}$ & $\begin{array}{c}\Delta \\
(\operatorname{arcsec})\end{array}$ & $\begin{array}{c}\mu \Delta t \\
(\operatorname{arcsec})\end{array}$ & $\begin{array}{c}\mu_{\alpha}^{*} \\
\left(\operatorname{mas~yr}^{-1}\right)\end{array}$ & $\begin{array}{c}\mu_{\delta} \\
\left(\operatorname{mas~yr}^{-1}\right)\end{array}$ \\
\hline 4016 & $\mathrm{BC}$ & 270.4 & 17.00 & 1998.97 & 267.2 & 15.66 & 1.6 & 1.2 & 62 & -79 \\
\hline 4878 & $\mathrm{AC}$ & 147.0 & 32.16 & 2000.79 & 145.3 & 32.42 & 1.0 & 0.8 & 66 & -23 \\
\hline 5276 & $\mathrm{AB}$ & 335.4 & 6.17 & 1999.97 & 338.0 & 5.32 & 0.9 & 1.6 & 118 & -55 \\
\hline 5313 & $\mathrm{AB}$ & 244.7 & 26.24 & 1998.79 & 245.0 & 25.99 & 0.3 & 1.0 & -79 & -2 \\
\hline 7845 & $\mathrm{AC}$ & 175.3 & 24.84 & 2000.01 & 174.6 & 24.44 & 0.5 & 0.0 & -2 & 2 \\
\hline 12062 & $\mathrm{AB}$ & 207.8 & 14.47 & 2003.60 & 199.4 & 14.09 & 4.2 & 2.9 & 230 & -83 \\
\hline 12067 & $\mathrm{AB}$ & 169.0 & 5.71 & 2000.89 & 172.4 & 5.25 & 0.6 & 1.5 & 97 & -76 \\
\hline 12685 & $\mathrm{AB}$ & 211.1 & 21.67 & 1997.77 & 212.2 & 21.26 & 0.6 & 0.3 & -22 & 4 \\
\hline 13336 & $\mathrm{AC}$ & 81.8 & 15.20 & 2000.75 & 92.3 & 20.79 & 6.5 & 6.8 & 487 & -283 \\
\hline 18218 & $\mathrm{AC}$ & 241.6 & 25.91 & 1999.78 & 241.4 & 26.18 & 0.3 & 1.0 & -43 & -67 \\
\hline 19389 & $\mathrm{CD}$ & 294.3 & 9.58 & 1999.76 & 284.2 & 9.39 & 1.7 & 1.6 & -25 & -126 \\
\hline 21099 & $\mathrm{AB}$ & 310.3 & 18.80 & 1999.83 & 310.9 & 17.41 & 1.4 & 1.3 & 102 & -41 \\
\hline 23396 & $\mathrm{AC}$ & 242.8 & 38.41 & 1997.84 & 242.1 & 40.04 & 1.7 & 1.3 & -63 & -92 \\
\hline 24016 & $\mathrm{BC}$ & 106.2 & 26.15 & 1997.93 & 103.9 & 27.32 & 1.6 & 0.7 & 58 & -5 \\
\hline 27067 & $\mathrm{AC}$ & 151.9 & 11.10 & 2000.86 & 154.2 & 12.07 & 1.1 & 1.1 & 20 & -85 \\
\hline 27246 & $\mathrm{AC}$ & 68.5 & 10.85 & 2000.23 & 67.9 & 10.93 & 0.1 & 2.8 & 142 & -187 \\
\hline 34110 & $\mathrm{AC}$ & 10.6 & 15.71 & 1998.94 & 8.3 & 15.23 & 0.8 & 0.7 & -50 & -20 \\
\hline 35265 & $\mathrm{BC}$ & 126.6 & 17.64 & 1999.98 & 135.4 & 18.43 & 2.9 & 2.2 & -58 & -173 \\
\hline 38018 & $\mathrm{AC}$ & 118.8 & 15.48 & 1999.08 & 127.1 & 15.66 & 2.3 & 2.1 & -93 & -151 \\
\hline 40298 & $\mathrm{BC}$ & 37.6 & 13.07 & 2000.17 & 39.2 & 15.53 & 2.5 & 2.2 & 142 & 113 \\
\hline 40479 & $\mathrm{AB}$ & 170.6 & 31.25 & 2000.07 & 169.1 & 32.09 & 1.2 & 0.9 & 71 & -15 \\
\hline 41319 & $\mathrm{BC}$ & 310.0 & 16.80 & 1997.85 & 298.4 & 18.15 & 3.8 & 2.9 & -192 & -152 \\
\hline 43426 & $\mathrm{CD}$ & 357.6 & 18.94 & 1999.85 & 350.9 & 20.72 & 2.9 & 2.5 & -173 & 113 \\
\hline 71843 & $\mathrm{BC}$ & 61.9 & 17.27 & 2000.20 & 61.7 & 16.38 & 0.9 & 0.7 & -58 & -18 \\
\hline 81608 & $\mathrm{BC}$ & 148.9 & 27.16 & 1999.27 & 151.9 & 28.53 & 2.0 & 1.2 & -28 & -98 \\
\hline 89207 & $\mathrm{AC}$ & 71.9 & 17.24 & 2000.54 & 72.9 & 16.51 & 0.8 & 0.5 & -1 & -39 \\
\hline 94905 & $\mathrm{AB}$ & 190.9 & 6.95 & 2000.32 & 192.7 & 6.68 & 0.3 & 0.4 & -29 & -10 \\
\hline 96395 & $\mathrm{AB}$ & 250.7 & 10.08 & 1997.47 & 249.3 & 10.06 & 0.2 & 2.4 & 0 & -200 \\
\hline 97222 & FK & 318.3 & 16.40 & 1998.33 & 304.1 & 13.05 & 6.5 & 5.3 & 13 & -441 \\
\hline 99965 & FL & 264.2 & 15.60 & 2000.37 & 257.3 & 18.70 & 3.7 & 3.7 & -221 & -216 \\
\hline 101430 & $\mathrm{AE}$ & 224.7 & 12.28 & 1998.48 & 224.4 & 9.12 & 3.2 & 2.5 & 154 & 140 \\
\hline 103641 & $\mathrm{DE}$ & 117.6 & 19.75 & 2000.36 & 108.4 & 21.29 & 3.6 & 3.8 & 232 & 209 \\
\hline 108473 & $\mathrm{AB}$ & 89.3 & 12.39 & 2000.75 & 89.5 & 12.37 & 0.1 & 3.4 & -248 & -133 \\
\hline 110291 & $\mathrm{AB}$ & 122.9 & 14.98 & 1998.78 & 131.5 & 13.62 & 2.5 & 2.4 & -190 & -64 \\
\hline 110291 & $\mathrm{AC}$ & 232.1 & 30.57 & 1998.78 & 233.1 & 33.69 & 3.2 & 2.4 & -190 & -64 \\
\hline 111148 & $\mathrm{BD}$ & 264.4 & 12.28 & 1998.78 & 268.3 & 14.59 & 2.5 & 2.2 & -176 & 34 \\
\hline 114456 & $\mathrm{CD}$ & 22.3 & 20.59 & 1999.76 & 23.7 & 16.84 & 3.8 & 3.6 & -86 & -286 \\
\hline 114456 & $\mathrm{CE}$ & 290.6 & 15.33 & 1999.76 & 273.6 & 15.74 & 4.6 & 3.6 & -86 & -286 \\
\hline 115655 & $\mathrm{BC}$ & 65.4 & 13.20 & 1998.80 & 66.8 & 13.81 & 0.7 & 0.8 & 47 & -45 \\
\hline
\end{tabular}

Note. The measurement for HIP 12062 comes from Zacharias et al. (2013), all others are 2MASS positions.

not known, the assumption of a $12 \mathrm{yr}$ time base is good enough for estimating the expected displacement.

\section{REFERENCES}

Africano, J. L., Cobb, C. L., Dunham, D. W., et al. 1975, AJ, 80, 689 Aitken, R. G. 1914, LicOB, 8, 93

Allen, P. R. 2007, ApJ, 668, 492

Alzner, A. 1998, A\&AS, 132, 253

Alzner, A. 2007, IAU Commission 26, Circular No., 163, 1

Andrade, M. 2007, RMxAA, 43, 237

Aristidi, E., Prieur, J.-L., Scardia, M., et al. 1999, A\&AS, 134, 545

Balega, I. I., Balega, Y. Y., Hoffmann, K.-H., et al. 2006, A\&A, 448, 703

Balega, Y. Y., Leushin, V. V., \& Weigelt, G. 2005, ARep, 49, 217

Baranec, C., Riddle, R., Law, N. M., et al. 2013, JVE, 72, e50021

Baranec, C., Riddle, R., Law, N. M., et al. 2014, ApJL, 790, L8

Bate, M. R. 2012, MNRAS, 419, 3115

Brendley, M., \& Mason, B. D. 2007, IAU Commission 26, Circular No., 163, 1 Burnham, S. W. 1873a, MNRAS, 34, 59
Burnham, S. W. 1873b, MNRAS, 33, 351

Burnham, S. W. 1894, PLicO, 2, 206

Couteau, P. 1990, A\&AS, 83, 331

Cutri, R. M., Skrutskie, M. F., van Dyk, S., et al. 2003, The IRSA 2MASS All-Sky Point Source Catalog. NASA/IPAC Infrared Science Archive

Cvetkovic, Z. 2010, IAU Commission 26, Circular No., 172, 1

Cvetkovic, Z. 2011, AJ, 141, 116

Cvetkovic, Z., \& Novakovic, B. 2006, Serbian AJ, 173, 73

Dawes, W. R. 1835, MemRAS, 8, 61

Dekany, R., Roberts, J., Burruss, R., et al. 2013, ApJ, 776, 130

de Lalande, J.-J. 1831, MmRAS, 4, 165

Docobo, J. A., \& Ling, J. F. 2008, IAU Commission 26, Circular No., 165, 1 Docobo, J. A., \& Ling, J. F. 2009, AJ, 138, 1159

Docobo, J. A., \& Ling, J. F. 2010, IAU Commission 26, Circular No., 172, 1

Docobo, J. A., \& Ling, J. F. 2011, IAU Commission 26, Circular No., 174, 1

Duchêne, G., \& Kraus, A. 2013, ARAA, 51, 269

Eggenberger, P., Miglio, A., Carrier, F., Fernandes, J., \& Santos, N. C. 2008, A\&A, 482, 631

Eggenberger, A., Udry, S., Chauvin, G., et al. 2007, A\&A, 474, 273

Espin, T. E. 1908, MNRAS, 68, 202 
Fekel, F. C., Scarfe, C. D., Barlow, D. J., et al. 1997, AJ, 113, 1095 Fuhrmann, K. 2004, AN, 325, 3

Ginski, C., Mugrauer, M., Seeliger, M., \& Eisenbeiss, T. 2012, MNRAS, 421,2498

Gorynya, N. A., \& Tokovinin, A. 2014, MNRAS, 441, 2316

Griffin, R. F. 2002, Obs, 121, 221

Griffin, R. F. 2012, JAA, 33, 29

Griffin, R. F., \& Heintz, W. D. 1987, JRASC, 81, 3

Griffin, R. F., \& Suchkov, A. A. 2003, ApJS, 147, 103

Hale, A. 1994, AJ, 107, 306

Harrington, R. S. 1992, in ASP Conf. Ser. 32, Complementary Approaches to Double and Multiple Star Research, ed. H. A. McAlister \& W. I. Hartkopf (IAU Colloq. 135; San Francisco, CA: ASP), 212

Hartkopf, W. I., \& Mason, B. D. 2000, IAU Commission 26, Circular No., 142,1

Hartkopf, W. I., \& Mason, B. D. 2009, AJ, 138, 813

Hartkopf, W. I., \& Mason, B. D. 2010, IAU Commission 26, Circular No., 170,1

Hartkopf, W. I., \& Mason, B. D. 2011a, AJ, 142, 56

Hartkopf, W. I., \& Mason, B. D. 2011b, IAU Commission 26, Circular No., 175,1

Hartkopf, W. I., \& Mason, B. D. 2013a, Catalog of Rectilinear Elements, USNO, http://ad.usno.navy.mil/wds/lin1.html

Hartkopf, W. I., \& Mason, B. D. 2013b, Sixth Catalog of Orbits of Visual Binary Stars, USNO, http://ad.usno.navy.mil/wds/orb6.html

Hartkopf, W. I., Mason, B. D., \& McAlister, H. A. 1996, AJ, 111, 370

Hartkopf, W. I., Mason, B. D., \& McAlister, H. A. 2001, AJ, 122, 3480

Hartkopf, W. I., Mason, B. D., \& Rafferty, T. 2008, AJ, 135, 1334

Hartkopf, W. I., Mason, B. D., \& Worley, C. E. 2001, AJ, 122, 3472

Hartkopf, W. I., McAlister, H. A., \& Franz, O. G. 1989, AJ, 98, 1014

Hartkopf, W. I., Tokovinin, A., \& Mason, B. D. 2012, AJ, 143, 42

Heintz, W. D. 1963, Veroff. Sternw. Munchen, 5, 247

Heintz, W. D. 1975, ApJS, 29, 331

Heintz, W. D. 1984, A\&AS, 56, 5

Heintz, W. D. 1986a, A\&AS, 64, 1

Heintz, W. D. 1986b, A\&AS, 65, 411

Heintz, W. D. 1988, A\&AS, 72, 543

Heintz, W. D. 1991, A\&AS, 90, 311

Heintz, W. D. 1994, AJ, 108, 2338

Heintz, W. D. 1995, ApJS, 99, 693

Heintz, W. D. 1996a, ApJS, 105, 475

Heintz, W. D. 1996b, AJ, 111, 408

Heintz, W. D. 1997, ApJS, 111, 335

Heintz, W. D. 1998, ApJS, 117, 587

Heintz, W. D. 2001, IAU Commission 26, Circular No., 143, 1

Henry, T. J., \& McCarthy, D. W. 1993, AJ, 106, 773

Hopmann, J. 1958, Mitt. Sternw. Wien, 9, 177

Horch, E. P., Bahi, L. A. P., Gaulin, J. R., et al. 2012, AJ, 143, 10

Horch, E. P., Robinson, S. E., Meyer, R. D., et al. 2002a, AJ, 123, 3442

Horch, E. P., Robinson, S. E., Ninkov, Z., et al. 2002b, AJ, 124, 2245

Hough, G. W. 1890, AJ, 9, 177

Janson, M., Hormuth, F., Bergfors, C., et al. 2012, ApJ, 754, 44

Jodar, E., Pérez-Garrido, A., Díaz-Sánchez, A., et al. 2013, MNRAS, 429,859

Kiyaeva, O. V., Kisselev, A. A., Polyakov, E. V., \& RafalŚkii, V. B. 2001, SvAL, 27, 391

Lang, K. R. 1992, Astrophysical Data. Planets and Stars (Berlin: Springer)

Law, N. M., Baranec, C., \& Riddle, R. 2014a, Proc. SPIE, Adaptive Optics Systems IV, 91480A (July 21, 2014); doi:10.1117/12.2056994

Law, N. M., Dhital, S., Kraus, A., Stassun, K. G., \& West, A. A. 2010, ApJ, 720,1727

Law, N. M., Morton, T., Baranec, C., et al. 2014b, ApJ, 791, 35

Lewis, T. 1900, MNRAS, 60, 494

Ling, J. F. 1992, AN, 313, 91

Ling, J. F. 2011, IAU Commission 26, Circular No., 174, 1

Ling, J. F. 2012, IAU Commission 26, Circular No., 176, 1

Lucy, L. B. 2006, A\&A, 457, 629

Makarov, V. V., \& Kaplan, G. H. 2005, AJ, 129, 2420

Mardling, R. A., \& Aarseth, S. J. 2001, MNRAS, 321, 398

Mason, B. D. 1996, AJ, 112, 2260

Mason, B. D., Douglass, G. G., \& Hartkopf, W. I. 1999, AJ, 117, 1023

Mason, B. D., \& Hartkopf, W. I. 2005, IAU Commission 26, Circular No., 156,1

Mason, B. D., \& Hartkopf, W. I. 2011, IAU Commission 26, Circular No., 173,1
Mason, B. D., \& Hartkopf, W. I. 2012, IAU Commission 26, Circular No., 178,1

Mason, B. D., Hartkopf, W. I., Gies, D. R., Henry, T. J., \& Helsel, J. W. 2009, AJ, 137,3358

Mason, B. D., Hartkopf, W. I., Holdenried, E. R., \& Rafferty, T. J. 2001a, AJ, 121,3224

Mason, B. D., Hartkopf, W. I., Wycoff, G. L., \& Rafferty, T. J. 2006, AJ, 131,2687

Mason, B. D., Hartkopf, W. I., Wycoff, G. L., et al. 2004a, AJ, 127, 539

Mason, B. D., Hartkopf, W. I., Wycoff, G. L., et al. 2004b, AJ, 128, 3012

Mason, B. D., McAlister, H. A., Hartkopf, W. I., \& Shara, M. M. 1995, AJ, 109,332

Mason, B. D., Wycoff, G. L., Hartkopf, W. I., Douglass, G. G., \& Worley, C. E. 2001b, AJ, 122, 3466

Metchev, S. A., \& Hillenbrand, L. A. 2009, ApJS, 181, 62

Muterspaugh, M. W., Hartkopf, W. I., Lane., B. F., et al. 2010a, AJ, 140,1623

Muterspaugh, M. W., Lane, B. F., Fekel, F. C., et al. 2008, AJ, 135, 766

Muterspaugh, M. W., Lane, B. F., Kulkarni, S. R., et al. 2010b, AJ, 140, 1657

Nordström, B., Mayor, M., Andersen, J, et al. 2004, A\&A, 418, 989

Perryman, M. A. C., \& ESA, 1997, in The Hipparcos and Tycho Catalogues, (ESA Publ. Ser. 1200; Noordwijk: ESA), Vol. 1

Popovic, G. M. 1969, BOBeo, 27, 33

Pourbaix, D., Tokovinin, A. A., Batten, A. H., et al. 2004, A\&A, 424, 727

Prieur, J.-L., Scardia, M., Pansecchi, L., Argyle, R. W., \& Sala, M. 2010, MNRAS, 407, 1913

Raghavan, D., McAlister, H. A., Henry, T. J., et al. 2010, ApJS, 190, 1

Rica, F. 2013, IAU Commission 26, Circular No., 181, 1

Riddle, R. L., Hogstrom, K., Papadopoulos, A., Baranec, C., \& Law, N. M. 2014, Proc. SPIE, 9152, 1

Roberts, L. C., Tokovinin, A., Riddle, R., Mason, B. D., \& Hartkopf, W. I. 2015, $\mathrm{AJ}$, in press

Rucinski, S. M., Pribulla, T., \& van Kerkwijk, M. H. 2007, AJ, 134, 2353

Scardia, M. 1981, AN, 302, 35

Scardia, M. 2001, IAU Commission 26, Circular No., 145, 2

Scardia, M., Argyle, R. W., Prieur, J.-L., et al. 2007, AN, 328, 146

Scardia, M., Prieur, J.-L., Koechlin, L., \& Aristidi, E. 2001, AN, 322, 161

Scardia, M., Prieur, J.-L., Koechlin, L., \& Aristidi, E. 2003, IAU Commission 26, Circular No., 151, 2

Scardia, M., Prieur, J.-L., Pansecchi, L., \& Argyle, R. W. 2009, IAU Commission 26, Circular No., 168, 1

Scardia, M., Prieur, J.-L., Pansecchi, L., \& Argyle, R. W. 2012, IAU Commission 26, Circular No., 177, 1

Scardia, M., Prieur, J.-L., Pansecchi, L., et al. 2008, AN, 329, 54

Seymour, D., \& Mason, B. 2000, IAU Commission 26, Circular No., 141, 2

Seymour, D., Mason, B. D., Hartkopf, W. I., \& Wycoff, G. L. 2002, AJ, 123,1023

Söderhjelm, S. 1999, A\&A, 341, 121

Starikova, G. A. 1978, SvAL, 4, 296

Starikova, G. A. 1983, SvAL, 9, 189

Struve, F. G. W. 1837, Mensurae Micrometricae Petropoli (see also 1837, AN 14, 249)

Terziev, E., Law, N. M., Arcavi, I., et al. 2013, ApJS, 206, 18

Tokovinin, A. 1997, A\&AS, 124, 75

Tokovinin, A. 2011, AJ, 141, 52

Tokovinin, A. 2012, AJ, 144, 56

Tokovinin, A. 2014, AJ, 147, 86

Tokovinin, A., Hartung, M., \& Hayward, T. L. 2010, AJ, 140, 510

Tokovinin, A., Hartung, M., \& Hayward, T. L. 2013, AJ, 146, 8

Tokovinin, A., Hartung, M., Hayward, T. L., \& Makarov, V. V. 2012, AJ, 144, 7

Tokovinin, A., \& Lépine, S. 2012, AJ, 144, 102

Tokovinin, A., Mason, B. D., \& Hartkopf, W. I. 2010, AJ, 139, 743

Tokovinin, A., Mason, B. D., \& Hartkopf, W. I. 2014, AJ, 147, 123

Tokovinin, A. A., \& Smekhov, M. G. 2002, A\&A, 382, 118

Tokovinin, A., Thomas, S., Sterzik, M., \& Udry, S. 2006, A\&A, 450, 681

Valbousquet, A. 1981, A\&AS, 45, 181

van Leeuwen, F. 2007, A\&A, 474, 653

Whitworth, A. P. 2001, in IAU Symp. 200, The Formation of Binary Stars, ed. H. Zinnecker \& R. D. Mathieu (San Francisco, CA: ASP)

York, D. G., Adelman, J., Anderson, Jr., et al. 2000, AJ, 120, 1579

Zacharias, N., Finch, C. T., Girard, T. M., et al. 2013, AJ, 145, 44

Zeller, G. 1965, Ann. Sternw. Wien, 26, 111

Zirm, H. 2008, IAU Commission 26, Circular No., 166, 1

Zirm, H. 2011, JDSO, 7, 24

Zulevic, D. J. 1997, BOBeo, 155, 109 